\title{
BOUNDARY INTEGRAL EQUATIONS FOR THE TRANSMISSION EIGENVALUE PROBLEM FOR MAXWELL'S EQUATIONS
}

\author{
FIORALBA CAKONI, HOUSSEM HADDAR AND SHIXU MENG
}

Communicated by Giovanni Monegato

\begin{abstract}
In this paper, we consider the transmission eigenvalue problem for Maxwell's equations corresponding to non-magnetic inhomogeneities with contrast in electric permittivity that changes sign inside its support. We formulate the transmission eigenvalue problem as an equivalent homogeneous system of the boundary integral equation and, assuming that the contrast is constant near the boundary of the support of the inhomogeneity, we prove that the operator associated with this system is Fredholm of index zero and depends analytically on the wave number. Then we show the existence of wave numbers that are not transmission eigenvalues which by an application of the analytic Fredholm theory implies that the set of transmission eigenvalues is discrete with positive infinity as the only accumulation point.
\end{abstract}

1. Introduction. The transmission eigenvalue problem is related to the scattering problem for an inhomogeneous media. In the current paper the underlying scattering problem is the scattering of electromagnetic waves by a (possibly anisotropic) non-magnetic material of bounded support $D$ situated in homogenous background, which in terms of the electric field reads:

$$
\begin{array}{ll}
\text { curl curl } \mathbf{E}^{s}-k^{2} \mathbf{E}^{s}=0 & \text { in } \mathbb{R}^{3} \backslash \bar{D} \\
\text { curl curl } \mathbf{E}-k^{2} N \mathbf{E}=0 & \text { in } D
\end{array}
$$

2010 AMS Mathematics subject classification. Primary 35J25, 45A05, 45C05, 45Q05, 78A25, 78A48.

Keywords and phrases. The transmission eigenvalue problem, inverse scattering, boundary integral equations, Maxwell's equations.

The research of the first author was supported in part by the Air Force Office of Scientific Research grant FA9550-13-1-0199 and NSF grant DMS-1515072. The research of the third author was supported in part by the Chateaubriand STEM fellowship.

Received by the editors on February 10, 2015. 


$$
\begin{array}{cc}
\nu \times \mathbf{E}=\nu \times \mathbf{E}^{s}+\nu \times \mathbf{E}^{i} & \text { on } \partial D \\
\nu \times \operatorname{curl} \mathbf{E}=\nu \times \operatorname{curl} \mathbf{E}^{s}+\nu \times \operatorname{curl} \mathbf{E}^{i} & \text { on } \partial D \\
\lim _{r \rightarrow \infty}\left(\operatorname{curl} \mathbf{E}^{s} \times x-i k r \mathbf{E}^{s}\right)=0 &
\end{array}
$$

where $\mathbf{E}^{i}$ is the incident electric field, $\mathbf{E}^{s}$ is the scattered electric field and

$$
N(x)=\frac{\epsilon(x)}{\epsilon_{0}}+i \frac{\sigma(x)}{\omega \epsilon_{0}}
$$

is the matrix index of refraction, $k=\omega \sqrt{\epsilon_{0} \mu_{0}}$ is the wave number corresponding to the background and the frequency $\omega$ and the SilverMüller radiation condition is satisfied uniformly with respect to $\widehat{x}=$ $x / r, r=|x|$. The difference $N-I$, in the following, is referred to as the contrast in the media. In scattering theory, transmission eigenvalues can be seen as an extension of the notion of resonant frequencies for impenetrable objects to the case of penetrable media. The transmission eigenvalue problem is related to non-scattering incident fields. Indeed, if $\mathbf{E}^{i}$ is such that $\mathbf{E}^{s}=0$, then $\left.\mathbf{E}\right|_{D}$ and $\mathbf{E}_{0}=\left.\mathbf{E}^{i}\right|_{D}$ satisfy the following homogenous problem

$$
\begin{array}{cl}
\text { curlcurl } \mathbf{E}-k^{2} N \mathbf{E}=0 & \text { in } D \\
\text { curlcurl } \mathbf{E}_{0}-k^{2} \mathbf{E}_{0}=0 & \text { in } D \\
\nu \times \mathbf{E}=\nu \times \mathbf{E}_{0} & \text { on } \partial D \\
\nu \times \operatorname{curl} \mathbf{E}=\nu \times \operatorname{curl} \mathbf{E}_{0} & \text { on } \partial D
\end{array}
$$

which is referred to as the transmission eigenvalue problem. Conversely, if (1.6)-(1.9) has a nontrivial solution $\mathbf{E}$ and $\mathbf{E}_{0}$ and $\mathbf{E}_{0}$ can be extended outside $D$ as a solution to curl curl $\mathbf{E}_{0}-k^{2} \mathbf{E}_{0}=0$, then if this extended $\mathbf{E}_{0}$ is considered as the incident field the corresponding scattered field is $\mathbf{E}^{s}=0$.

The transmission eigenvalue problem is a nonlinear and non-selfadjoint eigenvalue problem that is not covered by the standard theory of eigenvalue problems for elliptic equations. For a long time, research on the transmission eigenvalue problem mainly focused on showing that transmission eigenvalues form at most a discrete set, and we refer the reader to the survey paper [7] for the state of the art on this question up to 2010. From a practical point of view the question of discreteness was important to answer, since sampling methods for reconstructing the support of an inhomogeneous medium $[\mathbf{2}, \mathbf{3}]$ fail if the interrogat- 
ing frequency corresponds to a transmission eigenvalue. On the other hand, due to the non-selfadjointness of the transmission eigenvalue problem, the existence of transmission eigenvalues for non-spherically stratified media remained open for more than 20 years until Sylvester and Päivärinta [27] showed the existence of at least one transmission eigenvalue provided that the contrast in the medium is large enough. A full answer on the existence of transmission eigenvalues was given by Cakoni, Gintides and Haddar [5] where the existence of an infinite set of transmission eigenvalues was proven only under the assumption that the contrast in the medium does not change sign and is bounded away from zero (see also $[4,9,14,18]$ for Maxwell's equation). Since the appearance of these papers there has been an explosion of interest in the transmission eigenvalue problem, and the papers in the Special Issue of Inverse Problems on Transmission Eigenvalues, Volume 29, Number 10, October 2013, are representative of the myriad directions that this research has taken.

The discreteness and existence of transmission eigenvalues is very well understood under the assumption that the contrast does not change sign in all of $D$. Recently, for the scalar Helmholtz type equation, several papers have appeared that address both the question of discreteness and existence of transmission eigenvalue assuming that the contrast is of one sign only in a neighborhood of the nonhomogeneity's boundary $\partial D,[\mathbf{1}, \mathbf{1 2}, \mathbf{1 5}, \mathbf{2 3}, \mathbf{2 4}, \mathbf{2 8}, \mathbf{2 9}]$. The picture is not the same for the transmission eigenvalue problem for the Maxwell's equation. The only result in this direction is the proof of discreteness of transmission eigenvalues in [10] for magnetic materials, i.e., when there is contrast in both the electric prematurity and magnetic permeability. The $T$-coercivity approach used in [10] does not apply to our problem (1.6)-(1.9), which mathematically has a different structure from the case of magnetic materials, and this paper is dedicated to studying the discreteness of transmission eigenvalues for the problem considered under weaker assumptions of $N-I$. Before specifying our assumptions and approach let us rigorously formulate our transmission eigenvalue problem.

Formulation of the problem. Let $D \in \mathbb{R}^{3}$ be a bounded open and connected region with $C^{2}$-smooth boundary $\partial D:=\Gamma$ (we call it $\Gamma$ for convenience of notation as will be seen later), and let $\nu$ denote the outward unit normal vector on $\Gamma$. In general, we consider a $3 \times 3$ matrix- 
valued function $N$ with $L^{\infty}(D)$ entries such that $\bar{\xi} \cdot \operatorname{Re}(N) \xi \geq \alpha>0$ and $\bar{\xi} \cdot \operatorname{Im}(N) \xi \geq 0$ in $D$ for every $\xi \in \mathbb{C}^{3},|\xi|=1$. The transmission eigenvalue problem can be formulated as finding $\mathbf{E}, \mathbf{E}_{0} \in \mathbf{L}^{2}(D)$, $\mathbf{E}-\mathbf{E}_{0} \in \mathbf{H}_{0}\left(\operatorname{curl}^{2}, D\right)$ that satisfy

$$
\begin{array}{cc}
\text { curl curl } \mathbf{E}-k^{2} N \mathbf{E}=0 & \text { in } D \\
\text { curl curl } \mathbf{E}_{0}-k^{2} \mathbf{E}_{0}=0 & \text { in } D \\
\nu \times \mathbf{E}=\nu \times \mathbf{E}_{0} & \text { on } \Gamma \\
\nu \times \operatorname{curl} \mathbf{E}=\nu \times \operatorname{curl} \mathbf{E}_{0} & \text { on } \Gamma,
\end{array}
$$

where

$$
\mathbf{L}^{2}(D):=\left\{\mathbf{u}: \mathbf{u}_{j} \in L^{2}(D), j=1,2,3\right\},
$$

$\mathbf{H}\left(\operatorname{curl}^{2}, D\right):=\left\{\mathbf{u}: \mathbf{u} \in \mathbf{L}^{2}(D), \operatorname{curl} \mathbf{u} \in \mathbf{L}^{2}(D)\right.$ and curl curl $\left.\mathbf{u} \in \mathbf{L}^{2}(D)\right\}$, $\mathbf{H}_{0}\left(\operatorname{curl}^{2}, D\right):=\left\{\mathbf{u}: \mathbf{u} \in \mathbf{H}\left(\operatorname{curl}^{2}, D\right), \gamma_{t} \mathbf{u}=0\right.$ and $\gamma_{t} \operatorname{curl} \mathbf{u}=0$ on $\left.\Gamma\right\}$.

Definition 1.1. Values of $k \in \mathbb{C}$ for which the (1.10)-(1.13) has a nontrivial solution $\mathbf{E}, \mathbf{E}_{0} \in \mathbf{L}^{2}(D), \mathbf{E}-\mathbf{E}_{0} \in \mathbf{H}_{0}\left(\operatorname{curl}^{2}, D\right)$ are called transmission eigenvalues.

It is well known $[\mathbf{5}, \mathbf{1 6}]$ that, if $\operatorname{Re}(N-I)$ has one sign in $D$ the transmission eigenvalues form at most a discrete set with $+\infty$ as the only possible accumulation point, and if in addition $\operatorname{Im}(N)=0$, there exists an infinite set of real transmission eigenvalues. Our main concern is to understand the structure of the transmission eigenvalue problem in the case when $\operatorname{Re}(N-I)$ changes sign inside $D$. More specifically, in this case, we show that the transmission eigenvalues form at most a discrete set using an equivalent integral equation formulation of the transmission eigenvalue problem following the boundary integral equations approach developed in [15]. The assumption on the real part of the contract $N-I$ that we need in our analysis will become more precise later in the paper, but, roughly speaking, in our approach we allow for $\operatorname{Re}(N-I)$ to change sign in a compact subset of $D$. To this end, in the next section, we consider the simplest case when the electric permittivity is constant, i.e., $N=n I$ with positive $n \neq 1$, for which we develop and analyze an equivalent system of integral equations formulation of the corresponding transmission eigenvalue problem. This system of integral equations will then be a building 
block to study the more general case of the electric permittivity $N$. We note that the extension to Maxwell's equations of the approach in [15] is not a trivial task due to the more peculiar mapping properties of the electromagnetic boundary integral operators, as it will become clear in the paper.

2. Boundary integral equations for constant electric permittivity. Let $n>0$ be a constant such that $n \neq 1$, and consider the problem of finding $\mathbf{E}, \mathbf{E}_{0} \in \mathbf{L}^{2}(D), \mathbf{E}-\mathbf{E}_{0} \in \mathbf{H}_{0}\left(\operatorname{curl}^{2}, D\right)$ that satisfy

$$
\begin{aligned}
\text { curl curl } \mathbf{E}-k^{2} n \mathbf{E}=0 & \text { in } D \\
\text { curl curl } \mathbf{E}_{0}-k^{2} \mathbf{E}_{0}=0 & \text { in } D \\
\nu \times \mathbf{E}=\nu \times \mathbf{E}_{0} & \text { on } \Gamma \\
\nu \times(\operatorname{curl} \mathbf{E})=\nu \times\left(\operatorname{curl} \mathbf{E}_{0}\right) & \text { on } \Gamma .
\end{aligned}
$$

In the following, we set $k_{1}:=k \sqrt{n}$. Before formulating the transmission eigenvalue problem as an equivalent system of boundary integral equations we recall several integral operators and study their mapping properties. To this end, let us define the Hilbert spaces of tangential fields defined on $\Gamma$ :

$$
\begin{aligned}
\mathbf{H}^{s_{1}, s_{2}}(\operatorname{div}, \Gamma) & :=\left\{\mathbf{u} \in \mathbf{H}_{t}^{s_{1}}(\Gamma), \operatorname{div}_{\Gamma} \mathbf{u} \in H^{s_{2}}(\Gamma)\right\}, \\
\mathbf{H}^{s_{1}, s_{2}}(\operatorname{curl}, \Gamma) & :=\left\{\mathbf{u} \in \mathbf{H}_{t}^{s_{1}}(\Gamma), \operatorname{curl}_{\Gamma} \mathbf{u} \in \mathbf{H}^{s_{2}}(\Gamma)\right\},
\end{aligned}
$$

endowed with the respective natural norms, where $\operatorname{curl}_{\Gamma}$ and $\operatorname{div}_{\Gamma}$ are the surface curl and divergence operator, respectively, and for later use $\nabla_{\Gamma}$ denotes the tangential gradient operator. (Note that the boldface indicate vector spaces of vector fields, whereas non-bold face indicate vector spaces of scalar fields.) If $\gamma_{\Gamma} \mathbf{u}=\nu \times(\mathbf{u} \times \nu)$ denotes the tangential trace of a vector field $\mathbf{u}$ on the boundary $\Gamma$, we define the boundary integral operators:

$$
\mathbf{T}_{k}(\mathbf{u}):=\frac{1}{k} \gamma_{\Gamma}\left(k^{2} \int_{\Gamma} \Phi_{k}(\cdot, \mathbf{y}) \mathbf{u}(\mathbf{y}) d s_{y}+\nabla_{\Gamma} \int_{\Gamma} \Phi_{k}(\cdot, \mathbf{y}) \operatorname{div}{ }_{\Gamma} \mathbf{u}(\mathbf{y}) d s_{y}\right),
$$

and

$$
\mathbf{K}_{k}(\mathbf{u}):=\gamma_{\Gamma}\left(\operatorname{curl} \int_{\Gamma} \Phi_{k}(\cdot, y) \mathbf{u}(\mathbf{y}) d s_{y}\right)
$$


where

$$
\Phi_{k}(x, y)=\frac{1}{4 \pi} \frac{e^{i k|x-y|}}{|x-y|}
$$

is the fundament solution of the Helmholtz equation $\Delta u+k^{2} u=0$. Referring to $[\mathbf{1 5}, \mathbf{2 5}]$ for the mapping properties of the single layer potential

$$
S_{k}(\varphi):=\int_{\Gamma} \Phi_{k}(\cdot, \mathbf{y}) \varphi(\mathbf{y}) d s_{y},
$$

with scalar densities $\varphi$, we have that the boundary integral operator

$$
\mathbf{S}_{k}(\mathbf{u})=\int_{\Gamma} \Phi_{k}(\cdot, \mathbf{y}) \mathbf{u}(\mathbf{y}) d s
$$

acting on vector fields $\mathbf{u}$ is bounded from $\mathbf{H}^{-1 / 2+s}(\Gamma)$ to $\mathbf{H}^{1 / 2+s}(\Gamma)$ for $-1 \leq s \leq 1$, and hence,

$$
\begin{array}{r}
\mathbf{T}_{k}: \mathbf{H}^{-1 / 2,-3 / 2}(\operatorname{div}, \Gamma) \longrightarrow \mathbf{H}^{-1 / 2,-3 / 2}(\operatorname{curl}, \Gamma) \\
\mathbf{K}_{k}: \mathbf{H}^{-3 / 2,-1 / 2}(\operatorname{div}, \Gamma) \longrightarrow \mathbf{H}^{-3 / 2,-1 / 2}(\operatorname{curl}, \Gamma)
\end{array}
$$

are bounded linear operators. Now, from the Stratton-Chu formula [11] we have that

$$
\begin{aligned}
\mathbf{E}_{0}(\mathbf{x})= & \operatorname{curl} \int_{\Gamma}\left(\mathbf{E}_{0} \times \nu\right)(\mathbf{y}) \Phi_{k}(\mathbf{x}, \mathbf{y}) d s_{y} \\
& +\int_{\Gamma}\left(\operatorname{curl} \mathbf{E}_{0} \times \nu\right)(\mathbf{y}) \Phi_{k}(\mathbf{x}, \mathbf{y}) d s_{y} \\
& +\frac{1}{k^{2}} \nabla \int_{\Gamma} \operatorname{div}_{\Gamma}\left(\operatorname{curl} \mathbf{E}_{0} \times \nu\right)(\mathbf{y}) \Phi_{k}(\mathbf{x}, \mathbf{y}) d s_{y} \quad \text { for } \mathbf{x} \in D,
\end{aligned}
$$

with a similar expression for $\mathbf{E}$ where $k$ is replaced by $k_{1}:=k \sqrt{n}$, and hence we have the integral expression for $\mathbf{E}-\mathbf{E}_{0}$. Note by taking the difference $\mathbf{E}-\mathbf{E}_{0}$ we have the corresponding kernel $\Phi_{k_{1}}(\mathbf{x}, \mathbf{y})-\Phi_{k}(\mathbf{x}, \mathbf{y})$ is a smooth function of $\mathbf{x}, \mathbf{y}$, and, approaching the boundary $\Gamma$ and noting $\mathbf{E} \times \nu=\mathbf{E}_{0} \times \nu$ and curl $\mathbf{E} \times \nu=\operatorname{curl} \mathbf{E}_{0} \times \nu$, we have

$$
\begin{aligned}
\gamma_{\Gamma}\left(\mathbf{E}-\mathbf{E}_{0}\right)= & \left(\mathbf{K}_{k}-\mathbf{K}_{k_{1}}\right)\left(\mathbf{E}_{0} \times \nu\right) \\
& +\left(\frac{1}{k} \mathbf{T}_{k}-\frac{1}{k_{1}} \mathbf{T}_{k_{1}}\right)\left(\operatorname{curl} \mathbf{E}_{0} \times \nu\right), \\
\gamma_{\Gamma} \operatorname{curl}\left(\mathbf{E}-\mathbf{E}_{0}\right)= & \left(\mathbf{K}_{k}-\mathbf{K}_{k_{1}}\right)\left(\operatorname{curl} \mathbf{E}_{0} \times \nu\right)
\end{aligned}
$$




$$
+\left(k \mathbf{T}_{k}-k_{1} \mathbf{T}_{k_{1}}\right)\left(\mathbf{E}_{0} \times \nu\right) .
$$

From the boundary conditions (2.3) and (2.4) we have $\gamma_{\Gamma}\left(\mathbf{E}-\mathbf{E}_{0}\right)=0$ and $\gamma_{\Gamma} \operatorname{curl}\left(\mathbf{E}-\mathbf{E}_{0}\right)=0$, i.e.,

$$
\begin{aligned}
\mathbf{K}_{k}\left(\mathbf{E}_{0} \times \nu\right)+ & \frac{1}{k} \mathbf{T}_{k}\left(\operatorname{curl} \mathbf{E}_{0} \times \nu\right) \\
& -\mathbf{K}_{k_{1}}(\mathbf{E} \times \nu)-\frac{1}{k_{1}} \mathbf{T}_{k_{1}}(\operatorname{curl} \mathbf{E} \times \nu)=0, \\
\mathbf{K}_{k}\left(\operatorname{curl} \mathbf{E}_{0} \times \nu\right)+ & k \mathbf{T}_{k}\left(\mathbf{E}_{0} \times \nu\right) \\
& -\mathbf{K}_{k_{1}}(\operatorname{curl} \mathbf{E} \times \nu)-k_{1} \mathbf{T}_{k_{1}}(\mathbf{E} \times \nu)=0 .
\end{aligned}
$$

Introducing $\mathbf{M}=\mathbf{E} \times \nu=\mathbf{E}_{0} \times \nu$ and $\mathbf{J}=\operatorname{curl} \mathbf{E} \times \nu=\operatorname{curl} \mathbf{E}_{0} \times \nu$, we arrive at the following homogeneous system of boundary integral equations:

$$
\left(\begin{array}{cc}
k_{1} \mathbf{T}_{k_{1}}-k \mathbf{T}_{k} & \mathbf{K}_{k_{1}}-\mathbf{K}_{k} \\
\mathbf{K}_{k_{1}}-\mathbf{K}_{k} & \frac{1}{k_{1}} \mathbf{T}_{k_{1}}-\frac{1}{k} \mathbf{T}_{k}
\end{array}\right)\left(\begin{array}{c}
\mathbf{M} \\
\mathbf{J}
\end{array}\right)=\left(\begin{array}{l}
0 \\
0
\end{array}\right)
$$

for the unknowns $\mathbf{M}$ and $\mathbf{J}$. Let us define

$$
\begin{aligned}
\mathbf{L}(k) & =:\left(\begin{array}{cc}
k_{1} \mathbf{T}_{k_{1}}-k \mathbf{T}_{k} & \mathbf{K}_{k_{1}}-\mathbf{K}_{k} \\
\mathbf{K}_{k_{1}}-\mathbf{K}_{k} & \frac{1}{k_{1}} \mathbf{T}_{k_{1}}-\frac{1}{k} \mathbf{T}_{k}
\end{array}\right) \\
& =\left(\begin{array}{cc}
k \sqrt{n} \mathbf{T}_{k \sqrt{n}}-k \mathbf{T}_{k} & \mathbf{K}_{k \sqrt{n}}-\mathbf{K}_{k} \\
\mathbf{K}_{k \sqrt{n}}-\mathbf{K}_{k} & \frac{1}{k \sqrt{n}} \mathbf{T}_{k \sqrt{n}}-\frac{1}{k} \mathbf{T}_{k}
\end{array}\right) .
\end{aligned}
$$

Note that, while the operator $\mathbf{K}_{k_{1}}-\mathbf{K}_{k}$ is a smoothing pseudodifferential operator of order 2 (see, e.g., $[\mathbf{1 5}, \mathbf{1 7}]$ ), the operators in the main diagonal have a mixed structure. Indeed, from the expressions

$$
\begin{aligned}
k_{1} \mathbf{T}_{k_{1}}-k \mathbf{T}_{k} & =\left(k_{1}^{2} \mathbf{S}_{k_{1}}-k^{2} \mathbf{S}_{k}\right)+\nabla_{\Gamma} \circ\left(S_{k_{1}}-S_{k}\right) \circ \operatorname{div}_{\Gamma} \\
\frac{1}{k_{1}} \mathbf{T}_{k_{1}}-\frac{1}{k} \mathbf{T}_{k} & =\left(\mathbf{S}_{k_{1}}-\mathbf{S}_{k}\right)+\nabla_{\Gamma} \circ\left(\frac{1}{k_{1}^{2}} S_{k_{1}}-\frac{1}{k^{2}} S_{k}\right) \circ \operatorname{div}_{\Gamma}
\end{aligned}
$$

where $S$ and $\mathbf{S}$ are defined by (2.7) and (2.8), respectively, we can see that these operators have different behavior component-wise. Hence, a more delicate analysis is called for to find the correct function spaces for $\mathbf{M}, \mathbf{J}$ and their dual spaces in order to analyze the mapping properties of the operator $\mathbf{L}(k)$. 
Lemma 2.1. The dual space of $\mathbf{H}^{-3 / 2,-1 / 2}(\operatorname{div}, \Gamma)$ is $\mathbf{H}^{-1 / 2,1 / 2}(\operatorname{curl}, \Gamma)$. For $\mathbf{u}^{t} \in \mathbf{H}^{-1 / 2,1 / 2}(\operatorname{curl}, \Gamma)$ and $\mathbf{u} \in \mathbf{H}^{-3 / 2,-1 / 2}(\operatorname{div}, \Gamma),\left\langle\mathbf{u}^{t}, \mathbf{u}\right\rangle$ is understood by duality with respect to $\mathbf{L}^{2}(\Gamma)$ as a pivot space.

Proof. For any tangential fields $\mathbf{u} \in \mathbf{H}^{-3 / 2,-1 / 2}(\operatorname{div}, \Gamma)$ and $\mathbf{u}^{t} \in$ $\mathbf{H}^{-1 / 2,1 / 2}(\operatorname{curl}, \Gamma)$, we consider the corresponding Helmholtz orthogonal decomposition

$$
\mathbf{u}=\overrightarrow{\operatorname{curl}}_{\Gamma} q+\nabla_{\Gamma} p, \quad \mathbf{u}^{t}=\overrightarrow{\operatorname{curl}}_{\Gamma} q^{t}+\nabla_{\Gamma} p^{t} .
$$

Since $\operatorname{div} \Gamma_{\Gamma} \mathbf{u}=\operatorname{div}_{\Gamma} \nabla_{\Gamma} p=\Delta_{\Gamma} p \in H^{-1 / 2}(\Gamma)$, we have by eigensystem expansion (e.g., [26]) that $\nabla_{\Gamma} p \in \mathbf{H}^{1 / 2}(\Gamma)$. Similarly, from the fact that $\operatorname{curl}_{\Gamma} \mathbf{u}^{t} \in \mathbf{H}^{1 / 2}(\Gamma)$, we obtain that $\overrightarrow{\operatorname{curl}}_{\Gamma} q^{t} \in \mathbf{H}^{3 / 2}(\Gamma)$. Now

$$
\begin{aligned}
\left\langle\mathbf{u}^{t}, \mathbf{u}\right\rangle & =\left\langle\overrightarrow{\operatorname{curl}}_{\Gamma} q^{t}+\nabla_{\Gamma} p^{t}, \overrightarrow{\operatorname{curl}}_{\Gamma} q+\nabla_{\Gamma} p\right\rangle \\
& =\left\langle\overrightarrow{\operatorname{curl}}_{\Gamma} q^{t}, \overrightarrow{\operatorname{curl}}_{\Gamma} q\right\rangle+\left\langle\nabla_{\Gamma} p, \nabla_{\Gamma} p^{t}\right\rangle .
\end{aligned}
$$

Hence, the right hand side is well defined in the sense of duality of $\mathbf{H}^{3 / 2}(\Gamma)-\mathbf{H}^{-3 / 2}(\Gamma)$ and $\mathbf{H}^{1 / 2}(\Gamma)-\mathbf{H}^{-1 / 2}(\Gamma)$, and thus $\mathbf{H}^{-1 / 2,1 / 2}(\operatorname{curl}, \Gamma)$ is in the dual space of $\mathbf{H}^{-3 / 2,-1 / 2}(\operatorname{div}, \Gamma)$.

Furthermore, if $\mathbf{u}^{t}=\overrightarrow{\operatorname{curl}}_{\Gamma} q^{t}+\nabla_{\Gamma} p^{t}$ is in the dual space of $\mathbf{H}^{-3 / 2,-1 / 2}(\operatorname{div}, \Gamma)$, then $\left\langle\mathbf{u}^{t}, \cdot\right\rangle$ is continuous and linear on

$$
\mathbf{H}^{-3 / 2,-1 / 2}(\operatorname{div}, \Gamma) \text {. }
$$

Then, for $\mathbf{u}=\overrightarrow{\operatorname{curl}}_{\Gamma} q$,

$$
\left\langle\mathbf{u}^{t}, \mathbf{u}\right\rangle=\left\langle\overrightarrow{\operatorname{curl}}_{\Gamma} q^{t}, \overrightarrow{\operatorname{curl}}_{\Gamma} q\right\rangle .
$$

Notice $\overrightarrow{\operatorname{curl}}_{\Gamma} q$ is only in $\mathbf{H}^{-3 / 2}(\Gamma)$, therefore by eigensystem analysis $\overrightarrow{\operatorname{curl}}_{\Gamma} q^{t} \in \mathbf{H}^{3 / 2}(\Gamma)$ and $\operatorname{curl}_{\Gamma} \overrightarrow{\operatorname{curl}}_{\Gamma} q^{t} \in H^{1 / 2}(\Gamma)$, i.e., $\operatorname{curl}_{\Gamma} \mathbf{u}^{t} \in$ $H^{1 / 2}(\Gamma)$. Now, for $\mathbf{u}=\nabla_{\Gamma} p$ where $\nabla_{\Gamma} p \in \mathbf{H}^{1 / 2}(\Gamma)$

$$
\left\langle\mathbf{u}^{t}, \mathbf{u}\right\rangle=\left\langle\nabla_{\Gamma} p^{t}, \nabla_{\Gamma} p\right\rangle .
$$

Then $\nabla_{\Gamma} p^{t} \in \mathbf{H}^{-1 / 2}(\Gamma)$. Therefore, $\mathbf{u}^{t} \in \mathbf{H}^{-1 / 2,1 / 2}(\operatorname{curl}, \Gamma)$. Now, we have proved the lemma.

In the following, the spaces $\mathbf{H}^{-3 / 2,-1 / 2}(\operatorname{div}, \Gamma)$ and $\mathbf{H}^{-1 / 2,1 / 2}(\operatorname{curl}, \Gamma)$ are considered dual to each other in the duality defined in Lemma 2.1. 
In the next lemma, we establish some mapping properties of the operator $\mathbf{L}(k)$ given by (2.12).

Lemma 2.2. For a fixed $k$, the linear operator

$$
\begin{aligned}
\mathbf{L}(k): \mathbf{H}_{t}^{-1 / 2}(\Gamma) \times \mathbf{H}^{-3 / 2,-1 / 2}(\operatorname{div}, \Gamma) & \\
& \longrightarrow \mathbf{H}_{t}^{1 / 2}(\Gamma) \times \mathbf{H}^{-1 / 2,1 / 2}(\operatorname{curl}, \Gamma)
\end{aligned}
$$

is bounded. Moreover, the family of operators $\mathbf{L}(k)$ depends analytically on $k \in \mathbb{C} \backslash \mathbb{R}_{-}$.

Proof. Let $\mathbf{E}, \mathbf{E}_{0} \in \mathbf{L}^{2}(D), \mathbf{E}-\mathbf{E}_{0} \in \mathbf{H}_{0}\left(\operatorname{curl}^{2}, D\right)$ be a solution to the transmission eigenvalue problem (2.1)-(2.4). Hence,

$$
\mathbf{M}=\mathbf{E} \times \nu \in \mathbf{H}_{t}^{-1 / 2}(\Gamma), \quad \mathbf{J}=\operatorname{curl} \mathbf{E} \times \nu \in \mathbf{H}_{t}^{-3 / 2}(\Gamma) .
$$

Noting that $\operatorname{div}_{\Gamma}(\operatorname{curl} \mathbf{E} \times \nu)=\operatorname{curl}_{\Gamma} \operatorname{curl} \mathbf{E}=\left.\operatorname{curl}^{2} \mathbf{E} \cdot \nu\right|_{\Gamma}$, we have that $\operatorname{div}_{\Gamma} \mathbf{J} \in \mathbf{H}_{t}^{-1 / 2}(\Gamma)$ and therefore $(\mathbf{M}, \mathbf{J}) \in \mathbf{H}_{t}^{-1 / 2}(\Gamma) \times$ $\mathbf{H}^{-3 / 2,-1 / 2}(\operatorname{div}, \Gamma)$. It is known from [15] that $\mathbf{S}_{k}, \mathbf{S}_{k_{1}}-\mathbf{S}_{k}$ and $\mathbf{K}_{k_{1}}-\mathbf{K}_{k}$ are smoothing operators of order 1, 3 and 2, respectively. Then, using (2.13), we have that the following operators are bounded:

$$
\begin{aligned}
k_{1} \mathbf{T}_{k_{1}}-k \mathbf{T}_{k}: & \mathbf{H}_{t}^{-1 / 2}(\Gamma) \longrightarrow \mathbf{H}_{t}^{1 / 2}(\Gamma) \\
K k b-\mathbf{K}_{k}: & \mathbf{H}_{t}^{-3 / 2}(\Gamma) \longrightarrow \mathbf{H}_{t}^{1 / 2}(\Gamma) \\
\frac{1}{k_{1}} \mathbf{T}_{k_{1}}-\frac{1}{k} \mathbf{T}_{k}: & \mathbf{H}^{-3 / 2,-1 / 2}(\operatorname{div}, \Gamma) \longrightarrow \mathbf{H}_{t}^{-1 / 2}(\Gamma)
\end{aligned}
$$

Moreover,

$$
\begin{aligned}
\operatorname{curl}_{\Gamma}\left(\left(\mathbf{K}_{k_{1}}\right.\right. & \left.\left.-\mathbf{K}_{k}\right) \mathbf{M}+\left(\frac{1}{k_{1}} \mathbf{T}_{k_{1}}-\frac{1}{k} \mathbf{T}_{k}\right) \mathbf{J}\right) \\
& =\operatorname{curl}_{\Gamma}\left(\mathbf{K}_{k_{1}}-\mathbf{K}_{k}\right) \mathbf{M}+\operatorname{curl}_{\Gamma}\left(\mathbf{S}_{k_{1}}-\mathbf{S}_{k}\right) \mathbf{J} \in \mathbf{H}_{t}^{1 / 2}(\Gamma),
\end{aligned}
$$

and hence

$$
\begin{aligned}
\left(k_{1} \mathbf{T}_{k_{1}}-k \mathbf{T}_{k}\right) \mathbf{M}+\left(\mathbf{K}_{k_{1}}-\mathbf{K}_{k}\right) \mathbf{J} & \in \mathbf{H}_{t}^{1 / 2}(\Gamma), \\
\left(\mathbf{K}_{k_{1}}-\mathbf{K}_{k}\right) \mathbf{M}+\left(\frac{1}{k_{1}} \mathbf{T}_{k_{1}}-\frac{1}{k} \mathbf{T}_{k}\right) \mathbf{J} & \in \mathbf{H}^{-1 / 2,1 / 2}(\operatorname{curl}, \Gamma) .
\end{aligned}
$$


Hence, $\mathbf{L}(k)$ is bounded. Note that, since every component of $\mathbf{L}(k)$ is analytic on $\mathbb{C} \backslash \mathbb{R}_{-}$, then $\mathbf{L}(k)$ is analytic on $\mathbb{C} \backslash \mathbb{R}_{-}$(recall that $\left.k_{1}=k \sqrt{n}\right)$.

We need the following lemma to show the equivalence between the transmission eigenvalue problem and the system of integral equations (2.11).

Lemma 2.3. Let $\Omega$ be any bounded open region in $\mathbb{R}^{3}$, and denote $\mathbf{V}\left(\operatorname{curl}^{2}, \Omega\right):=\left\{\mathbf{u}: \mathbf{u} \in \mathbf{L}^{2}(\Omega), \operatorname{curl}^{2} \mathbf{u} \in \mathbf{L}^{2}(\Omega)\right\}$. For $\varphi \in \mathbf{H}_{t}^{-1 / 2}(\Gamma)$, $\psi \in \mathbf{H}^{-(3 / 2),-(1 / 2)}(\operatorname{div}, \Gamma)$, we define

$$
\widetilde{\mathbf{M}}_{1}(\varphi)(\mathbf{x}):=\operatorname{curl} \int_{\Gamma} \Phi_{k}(\mathbf{x}, \mathbf{y}) \varphi(\mathbf{y}) d s_{y}, \quad x \in \mathbb{R}^{3} \backslash \Gamma,
$$

and

$$
\widetilde{\mathbf{M}}_{2}(\psi)(\mathbf{y}):=\int_{\Gamma} \Phi_{k}(\mathbf{x}, \mathbf{y}) \psi(\mathbf{y}) d s_{y}, \quad x \in \mathbb{R}^{3} \backslash \Gamma .
$$

Then $\widetilde{\mathbf{M}}_{1}$ is continuous from $\mathbf{H}_{t}^{-1 / 2}(\Gamma)$ to $\mathbf{V}\left(\operatorname{curl}^{2}, D^{ \pm}\right)$and $\widetilde{\mathbf{M}}_{2}$ is continuous from $\mathbf{H}^{-3 / 2,-1 / 2}(\operatorname{div}, \Gamma)$ to $\mathbf{V}\left(\operatorname{curl}^{2}, D^{ \pm}\right)$where $D^{-}=D$ and $D^{+}=B_{R} \backslash \bar{D}$ with a sufficient large ball $B_{R}$ containing the closure of $D$. Furthermore, the following jump relations hold:

$$
\begin{aligned}
{\left[\gamma_{t} \widetilde{\mathbf{M}}_{1}(\varphi)\right] } & =\varphi \quad \text { in } \mathbf{H}_{t}^{-1 / 2}(\Gamma), \\
{\left[\gamma_{t} \operatorname{curl} \widetilde{\mathbf{M}}_{1}(\varphi)\right] } & =0 \quad \text { in } \mathbf{H}_{t}^{-3 / 2}(\Gamma), \\
{\left[\gamma_{t} \operatorname{curl} \widetilde{\mathbf{M}}_{2}(\psi)\right] } & =\psi \quad \text { in } \mathbf{H}_{t}^{-3 / 2}(\Gamma), \\
{\left[\operatorname{div}_{\Gamma} \gamma_{t} \operatorname{curl} \widetilde{\mathbf{M}}_{2}(\psi)\right] } & =\operatorname{div}_{\Gamma} \psi \quad \text { in } H^{-1 / 2}(\Gamma) .
\end{aligned}
$$

Proof. Let us denote by $\langle\cdot, \cdot\rangle$ the $\mathbf{H}_{t}^{1 / 2}(\Gamma)-\mathbf{H}_{t}^{-1 / 2}(\Gamma)$ or $H^{1 / 2}(\Gamma)$ $H^{-1 / 2}(\Gamma)$ duality product. Since $\varphi \in \mathbf{H}_{t}^{-1 / 2}(\Gamma)$, then, from the classical results for single layer potentials,

$$
\left\|\widetilde{\mathbf{M}}_{1}(\varphi)\right\|_{\mathbf{L}^{2}\left(D^{ \pm}\right)} \leq c\left\|\int_{\Gamma} \Phi_{k}(\mathbf{x}, \mathbf{y}) \varphi(\mathbf{y}) d s_{y}\right\|_{\mathbf{H}^{1}\left(D^{ \pm}\right)} \leq c\|\varphi\|_{\mathbf{H}_{t}^{-1 / 2}(\Gamma)},
$$

and, since $\operatorname{curl}^{2} \widetilde{\mathbf{M}}_{1}(\varphi)-k^{2} \widetilde{\mathbf{M}}_{1}(\varphi)=0$ in $D^{ \pm}$, then

$$
\left\|\operatorname{curl}^{2} \widetilde{\mathbf{M}}_{1}(\varphi)\right\|_{\mathbf{L}^{2}\left(D^{ \pm}\right)}=\left|k^{2}\right|\left\|\widetilde{\mathbf{M}}_{1}(\varphi)\right\|_{\mathbf{L}^{2}\left(D^{ \pm}\right)}
$$




$$
\leq c\|\varphi\|_{\mathbf{H}_{t}^{-1 / 2}(\Gamma)}
$$

where $c$ is some constant depending on $k$. For $\psi \in \mathbf{H}^{-3 / 2,-1 / 2}(\operatorname{div}, \Gamma)$, we have from [15],

$$
\left\|\widetilde{\mathbf{M}}_{2}(\psi)\right\|_{\mathbf{L}^{2}\left(D^{ \pm}\right)} \leq c\|\psi\|_{\mathbf{H}_{t}^{-3 / 2}(\Gamma)} .
$$

Notice that

$$
\begin{aligned}
\operatorname{curl}^{2} \widetilde{\mathbf{M}}_{2}(\psi)(\mathbf{x})= & k^{2} \int_{\Gamma} \Phi_{k}(\mathbf{x}, \mathbf{y}) \psi(\mathbf{y}) d s_{y} \\
& +\nabla \int_{\Gamma} \operatorname{div} \Gamma \psi(\mathbf{y}) \Phi_{k}(\cdot, \mathbf{y}) d s_{y}
\end{aligned}
$$

and $\operatorname{div}{ }_{\Gamma} \psi \in H^{-1 / 2}(\Gamma)$; hence, we have from [15],

$$
\left\|\operatorname{curl}^{2} \widetilde{\mathbf{M}}_{2}(\psi)\right\|_{\mathbf{L}^{2}\left(D^{ \pm}\right)} \leq c\left(\|\psi\|_{\mathbf{H}_{t}^{-3 / 2}(\Gamma)}+\left\|\operatorname{div}{ }_{\Gamma} \psi\right\|_{H^{-1 / 2}(\Gamma)}\right) .
$$

This proves the continuity property of $\widetilde{\mathbf{M}}_{1}$ and $\widetilde{\mathbf{M}}_{2}$. To prove the jump relations, we will use a density argument. Let

$$
\mathbf{u}^{ \pm}=\operatorname{curl} \int_{\Gamma} \Phi_{k}(\mathbf{x}, \mathbf{y}) \varphi(\mathbf{y}) d s_{y}, \quad \text { in } D^{ \pm} .
$$

We define the tangential component $\gamma_{t} \mathbf{u}^{ \pm}$by duality. For $\alpha \in \mathbf{H}_{t}^{1 / 2}(\Gamma)$, $\|\alpha\|_{\mathbf{H}_{t}^{1 / 2}(\Gamma)}=1$, there exists $\mathbf{w}^{ \pm} \in \mathbf{H}^{2}\left(D^{ \pm}\right)$and $\mathbf{w}^{+}$compactly supported in $B_{R}$ such that $\gamma_{t} \operatorname{curl} \mathbf{w}=\alpha, \gamma_{t} \mathbf{w}=0$ and $\|\mathbf{w}\|_{\mathbf{H}^{2}\left(D^{ \pm}\right)} \leq$ $c\|\alpha\|_{\mathbf{H}_{t}^{1 / 2}(\Gamma)}($ see $[\mathbf{1 6}])$. Moreover,

$$
\left\langle\alpha, \gamma_{\mathbf{t}} \mathbf{u}^{ \pm}\right\rangle= \pm \int_{D^{ \pm}}\left(\mathbf{u}^{ \pm} \cdot \operatorname{curl}^{2} \mathbf{w}^{ \pm}-\mathbf{w}^{ \pm} \cdot \operatorname{curl}^{2} \mathbf{u}^{ \pm}\right) d \mathbf{x}
$$

Then

$$
\begin{aligned}
\left|\left\langle\alpha, \gamma_{t} \mathbf{u}^{ \pm}\right\rangle\right| & \leq\left(\|\mathbf{u}\|_{\mathbf{L}^{2}\left(D^{ \pm}\right)}+\left\|\operatorname{curl}^{2} \mathbf{u}\right\|_{\mathbf{L}^{2}\left(D^{ \pm}\right)}\right)\|\mathbf{w}\|_{\mathbf{H}^{2}\left(D^{ \pm}\right)} \\
& \leq c_{1}\left(\|\mathbf{u}\|_{\mathbf{L}^{2}\left(D^{ \pm}\right)}+\left\|\operatorname{curl}^{2} \mathbf{u}\right\|_{\mathbf{L}^{2}\left(D^{ \pm}\right)}\right) \\
& \leq c_{2}\|\varphi\|_{\mathbf{H}_{t}^{-1 / 2}(\Gamma)}
\end{aligned}
$$

where $c_{1}$ and $c_{2}$ are independent from $u$; therefore $\left\|\gamma_{t} \mathbf{u}^{ \pm}\right\|_{\mathbf{H}_{t}^{-1 / 2}(\Gamma)} \leq$ $c_{2}\|\varphi\|_{\mathbf{H}_{t}^{-1 / 2}(\Gamma)}$. Choosing $\varphi_{n} \in \mathbf{H}^{-(1 / 2),-(1 / 2)}(\operatorname{div}, \Gamma)$ such that $\varphi_{n} \rightarrow \varphi$ 
in $\mathbf{H}_{t}^{-1 / 2}(\Gamma)$ yields

$$
\left\|\gamma_{t} \mathbf{u}^{ \pm}-\gamma_{t} \mathbf{u}_{n}^{ \pm}\right\|_{\mathbf{H}_{t}^{-1 / 2}(\Gamma)} \leq c\left\|\varphi-\varphi_{n}\right\|_{\mathbf{H}_{t}^{-1 / 2}(\Gamma)} \longrightarrow 0,
$$

since $\left[\gamma_{t} \mathbf{u}_{n}\right]=\varphi_{\mathbf{n}}$ for $\varphi_{n} \in \mathbf{H}^{-1 / 2,-1 / 2}(\operatorname{div}, \Gamma)$ (see [26]). Letting $n \rightarrow \infty$ yields $\left[\gamma_{t} \mathbf{u}\right]=\varphi$ in $\mathbf{H}_{t}^{-1 / 2}(\Gamma)$; hence, (2.15) holds. In a similar argument, we can prove (2.16) and (2.17).

From (2.17), we have

$$
\left[\gamma_{t} \operatorname{curl} \widetilde{\mathbf{M}}_{2}(\psi)\right]=\psi \quad \text { in } \mathbf{H}_{t}^{-3 / 2}(\Gamma) .
$$

Then

$$
\left[\operatorname{div}_{\Gamma} \gamma_{t} \operatorname{curl} \widetilde{\mathbf{M}}_{2}(\psi)\right]=\operatorname{div}_{\Gamma} \psi
$$

in the distributional sense. Notice $\operatorname{div}_{\Gamma} \psi$ and $\left(\operatorname{div}_{\Gamma} \gamma_{t} \operatorname{curl} \widetilde{\mathbf{M}}_{2}(\psi)\right)^{ \pm}$are in $H^{-1 / 2}(\Gamma)$. Then (2.18) holds.

Now we are ready to prove equivalence between the transmission eigenvalue problem and the system of integral equations (2.11). Our proof follows the lines of the proof of [15, Theorem 2.2].

Theorem 2.4. The following statements are equivalent:

(i) There exist non trivial $\mathbf{E}, \mathbf{E}_{0} \in \mathbf{L}^{2}(D), \mathbf{E}-\mathbf{E}_{0} \in \mathbf{H}\left(\mathrm{curl}^{2}, D\right)$ such that (2.1)-(2.4) holds.

(ii) There exists non trivial $(\mathbf{M}, \mathbf{J}) \in \mathbf{H}_{t}^{-1 / 2}(\Gamma) \times \mathbf{H}^{-3 / 2,-1 / 2}(\operatorname{div}, \Gamma)$ such that (2.11) holds and either $\mathbf{E}_{0}^{\infty}(\mathbf{M}, \mathbf{J})=0$ or $\mathbf{E}^{\infty}(\mathbf{M}, \mathbf{J})=$ 0 , where

$$
\begin{aligned}
\mathbf{E}_{0}^{\infty}(\mathbf{M}, \mathbf{J})(\widehat{x})= & \widehat{x} \times\left(\frac{1}{4 \pi} \operatorname{curl} \int_{\Gamma} \mathbf{M}(y) e^{-i k \hat{x} \cdot y} d s_{y}\right. \\
& \left.+\frac{1}{4 \pi k^{2}} \nabla \int_{\Gamma} \operatorname{div}_{\Gamma} \mathbf{J}(y) e^{-i k \hat{x} \cdot y} d s_{y}+\int_{\Gamma} \mathbf{J}(y) e^{-i k \hat{x} \cdot y} d s_{y}\right) \times \widehat{x},
\end{aligned}
$$

with the same expression for $\mathbf{E}^{\infty}(\mathbf{M}, \mathbf{J})$ where $k$ is replaced by $k_{1}$.

Proof. Assume (i) holds. Then, from the argument above (2.11) we have that $\mathbf{M}$ and $\mathbf{J}$ satisfy (2.11), and hence it suffices to show $\mathbf{E}_{0}^{\infty}(\mathbf{M}, \mathbf{J})=0$ and $\mathbf{E}^{\infty}(\mathbf{M}, \mathbf{J})=0$. To this end, recall that $\mathbf{E}_{0}$ has the 
following representation:

$$
\begin{aligned}
\mathbf{E}_{0}(x)= & \operatorname{curl} \int_{\Gamma} \mathbf{M}(y) \Phi_{k}(x, y) d s_{y}+\int_{\Gamma} \mathbf{J}(y) \Phi_{k}(\cdot, y) d s_{y} \\
& +\frac{1}{k^{2}} \nabla \int_{\Gamma} \operatorname{div}_{\Gamma} \mathbf{J}(y) \Phi_{k}(\cdot, y) d s_{y}
\end{aligned}
$$

where $\mathbf{E}_{0} \times \nu=\mathbf{E} \times \nu=\mathbf{M}$ and $\operatorname{curl} \mathbf{E}_{0} \times \nu=\operatorname{curl} \mathbf{E} \times \nu=\mathbf{J}$. Then, from the jump relations $(2.15)-(2.18)$ of the vector potentials applied to $(2.20)$ and $(2.11)$ (see also $[\mathbf{1 5}]$ ), we obtain that $\left(\mathbf{E}_{0} \times \nu\right)^{+}=0$, $\left(\operatorname{curl} \mathbf{E}_{0} \times \nu\right)^{+}=0(+$ denotes the traces from outside of $D)$, and hence the far field pattern $\mathbf{E}_{0}^{\infty}(\mathbf{M}, \mathbf{J})$ varnishes. The asymptotic expression of the fundamental solution $\Phi(\cdot, \cdot)$ in [11, page 23] yields (2.19). Similarly, we can prove that $\mathbf{E}^{\infty}(\mathbf{M}, \mathbf{J})=0$.

Next, assume that (2) holds and define

$$
\begin{aligned}
\mathbf{E}_{0}(x)= & \operatorname{curl} \int_{\Gamma} \mathbf{M}(y) \Phi_{k}(x, y) d s_{y}+\int_{\Gamma} \mathbf{J}(y) \Phi_{k}(\cdot, y) d s_{y} \\
& +\frac{1}{k^{2}} \nabla \int_{\Gamma} \operatorname{div}_{\Gamma} \mathbf{J}(y) \Phi_{k}(\cdot, y) d s_{y}, \quad x \in \mathbb{R}^{3} \backslash \Gamma,
\end{aligned}
$$

with the same expression for $\mathbf{E}$ where $k$ is replaced by $k_{1}$. Again, from the jump relations of vector potentials and (2.11), we have

$$
\begin{aligned}
& \text { curlcurl } \mathbf{E}-k^{2} n \mathbf{E}=0, \quad \text { curlcurl } \mathbf{E}_{0}-k^{2} \mathbf{E}_{0}=0 \quad \text { in } D \\
& \mathbf{E} \times \nu=\mathbf{E}_{0} \times \nu, \quad \operatorname{curl} \mathbf{E} \times \nu=\operatorname{curl} \mathbf{E}_{0} \times \nu \quad \text { on } \Gamma
\end{aligned}
$$

(note that $\mathbf{E}$ and $\mathbf{E}_{0}$ are in $L^{2}(D)$. Therefore, it suffices to show $\mathbf{E}_{0}$ and $\mathbf{E}$ are non trivial. Assume, to the contrary, that $\mathbf{E}_{0}=\mathbf{E}=0$, and, without loss of generality, $\mathbf{E}^{\infty}(\mathbf{M}, \mathbf{J})=0$. Then, by Rellich's lemma (see, e.g., [11]) $\mathbf{E}=0$ in $\mathbb{R}^{3} \backslash \bar{D}$. Hence, the jump relations imply $\mathbf{M}=0$ and $\mathbf{J}=0$, which is a contradiction to the assumptions in (ii). This proves the theorem.

The above discussion allows us to conclude that, in order to prove the discreteness of the transmission eigenvalues, we need to show that the kernel of the operator $\mathbf{L}(k)$ is non-trivial for at most a discrete set of wave numbers $k$.

2.1. Properties of the operator $\mathbf{L}(k)$. In the following, we will show the operator $\mathbf{L}(k)$ is Fredholm of index zero and use the analytic 
Fredholm theory to obtain our main theorem. To this end, we first show that, for the purely complex wave number $k:=i \kappa, \kappa>0, \mathbf{L}(k)$ restricted to

$$
\mathbf{H}_{0}^{-3 / 2,-1 / 2}(\operatorname{div}, \Gamma):=\left\{\mathbf{u} \in \mathbf{H}^{-3 / 2,-1 / 2}(\operatorname{div}, \Gamma), \operatorname{div}_{\Gamma} \mathbf{u}=0\right\} .
$$

satisfies the coercive property. In the following lemma, we use the shorthand notation $\mathbf{H}_{0}(\Gamma):=\mathbf{H}_{t}^{-1 / 2}(\Gamma) \times \mathbf{H}_{0}^{-3 / 2,-1 / 2}(\operatorname{div}, \Gamma)$ and its dual space $\mathbf{H}^{*}(\Gamma):=\mathbf{H}_{t}^{1 / 2}(\Gamma) \times\left(\mathbf{H}_{0}^{-3 / 2,-1 / 2}(\operatorname{div}, \Gamma)\right)^{\prime}$ where the dual $\left(\mathbf{H}_{0}^{-3 / 2,-1 / 2}(\operatorname{div}, \Gamma)\right)^{\prime}$ of the subspace $\mathbf{H}_{0}^{-3 / 2,-1 / 2}(\operatorname{div}, \Gamma) \subset$ $\mathbf{H}^{-3 / 2,-1 / 2}(\operatorname{div}, \Gamma)$ is understood in the sense of the duality defined by Lemma 2.1 .

Lemma 2.5. Let $\kappa>0$. The operator $\mathbf{L}(i \kappa): \mathbf{H}_{0}(\Gamma) \rightarrow \mathbf{H}^{*}(\Gamma)$ is strictly coercive, i.e.,

$$
\begin{aligned}
\left|\left\langle\mathbf{L}(i \kappa)\left(\begin{array}{c}
\mathbf{M} \\
\mathbf{J}
\end{array}\right),\left(\begin{array}{c}
\mathbf{M} \\
\mathbf{J}
\end{array}\right)\right\rangle\right| \\
\quad \geq c\left(\|\mathbf{M}\|_{\mathbf{H}_{t}^{-1 / 2}(\Gamma)}+\|\mathbf{J}\|_{\mathbf{H}^{-3 / 2,-1 / 2}(\operatorname{div}, \Gamma)}\right),
\end{aligned}
$$

where $c$ is a constant depending only on $\kappa$.

Proof. We consider the following problem. For given $(\mathbf{M}, \mathbf{J}) \in$ $\mathbf{H}_{t}^{-1 / 2}(\Gamma) \times \mathbf{H}_{0}^{-3 / 2,-1 / 2}(\operatorname{div}, \Gamma)$ find $\mathbf{U} \in \mathbf{L}^{2}\left(\mathbb{R}^{3}\right)$, curl $\mathbf{U} \in \mathbf{L}^{2}\left(\mathbb{R}^{3}\right)$, $\operatorname{curl}^{2} \mathbf{U} \in \mathbf{L}^{2}\left(\mathbb{R}^{3}\right)$ such that

$$
\begin{array}{rc}
\left(\operatorname{curl}^{2}+n \kappa^{2}\right)\left(\operatorname{curl}^{2}+\kappa^{2}\right) \mathbf{U}=0 & \text { in } \mathbb{R}^{3} \backslash \Gamma \\
{\left[\nu \times \operatorname{curl}^{2} \mathbf{U}\right]=\left(n \kappa^{2}-\kappa^{2}\right) \mathbf{M}} & \text { on } \Gamma \\
{\left[\nu \times \operatorname{curl}^{3} \mathbf{U}\right]=\left(n \kappa^{2}-\kappa^{2}\right) \mathbf{J}} & \text { on } \Gamma
\end{array}
$$

where [.] denotes the jump across $\Gamma$. Multiplying (2.21) by a test function $\mathbf{W}$ and integrating by parts yields

$$
\begin{aligned}
\int_{\mathbb{R}^{3} \backslash \Gamma}\left(\operatorname{curl}^{2}+n \kappa^{2}\right) \mathbf{U} \cdot\left(\operatorname{curl}^{2}+\kappa^{2}\right) \overline{\mathbf{W}} d x \\
\quad=\left(n \kappa^{2}-\kappa^{2}\right)\left(\int_{\Gamma} \gamma_{\Gamma} \operatorname{curl} \overline{\mathbf{W}} \cdot \mathbf{M} d s+\int_{\Gamma} \gamma_{\Gamma} \overline{\mathbf{W}} \cdot \mathbf{J} d s\right)
\end{aligned}
$$

First we show that the right hand side is well defined. Note that $\operatorname{div}(\operatorname{curl} \mathbf{W})=0$; hence, from $[\mathbf{2 6}], \operatorname{curl} \mathbf{W} \in \mathbf{H}^{1}\left(\mathbb{R}^{3}\right)$, and thus 
$\gamma_{\Gamma} \operatorname{curl} \mathbf{W} \in \mathbf{H}_{t}^{1 / 2}(\Gamma)$, which implies $\int_{\Gamma} \gamma_{\Gamma} \operatorname{curl} \overline{\mathbf{W}} \cdot \mathbf{M} d s$ is defined in $\mathbf{H}_{t}^{1 / 2}(\Gamma), \mathbf{H}_{t}^{-1 / 2}(\Gamma)$ duality. Since $\gamma_{\Gamma} \mathbf{W} \in \mathbf{H}_{t}^{-1 / 2}(\Gamma)$ and $\operatorname{curl}_{\Gamma} \mathbf{W}=$ $\gamma_{\Gamma}$ curl $\mathbf{W} \in \mathbf{H}_{t}^{1 / 2}(\Gamma)$, then, from Lemma $2.1, \int_{\Gamma} \gamma_{\Gamma} \overline{\mathbf{W}} \cdot \mathbf{J} d s$ is well defined.

Now let

$$
\mathbf{V}:=\left\{\mathbf{U} \in \mathbf{L}^{2}\left(\mathbb{R}^{3}\right), \quad \operatorname{curl} \mathbf{U} \in \mathbf{L}^{2}\left(\mathbb{R}^{3}\right), \quad \operatorname{curl}^{2} \mathbf{U} \in \mathbf{L}^{2}\left(\mathbb{R}^{3}\right)\right\}
$$

equipped with the norm

$$
\|\mathbf{U}\|_{\mathbf{V}}^{2}=\int_{\mathbb{R}^{3}}\left(\left|\operatorname{curl}^{2} \mathbf{U}\right|^{2}+|\operatorname{curl} \mathbf{U}|^{2}+|\mathbf{U}|^{2}\right) d x .
$$

Next, taking $\mathbf{W}=\mathbf{U}$ in the continuous sesquilinear form in the lefthand side of (2.24) and, after integrating by parts (note that $\mathbf{U}$ and curl $\mathbf{U}$ are continuous across $\Gamma$, we obtain

$$
\begin{aligned}
& \int_{\mathbb{R}^{3} \backslash \Gamma}\left(\operatorname{curl}^{2}+n \kappa^{2}\right) \mathbf{U} \cdot\left(\operatorname{curl}^{2}+\kappa^{2}\right) \overline{\mathbf{U}} d x \\
& =\int_{\mathbb{R}^{3}}\left(\left|\operatorname{curl}^{2} \mathbf{U}\right|^{2}+\left(n \kappa^{2}+\kappa^{2}\right)|\operatorname{curl} \mathbf{U}|^{2}+n \kappa^{2} \kappa^{2}|\mathbf{U}|^{2}\right) d x \geq c\|\mathbf{U}\|_{\mathbf{V}},
\end{aligned}
$$

where $c$ is a constant depending on $\kappa$. The Lax-Milgram lemma guaranties the existence of a unique solution to (2.24). Up until here we did not need that $\operatorname{div}_{\Gamma} \mathbf{J}=0$. Next, we define

$$
\begin{aligned}
\mathbf{U}= & \operatorname{curl} \int_{\Gamma} \mathbf{M}(y)\left(\Phi_{\sqrt{n} \kappa}(\cdot, y)-\Phi_{\kappa}(\cdot, y)\right) d s \\
& +\int_{\Gamma} \mathbf{J}(y)\left(\Phi_{\sqrt{n} \kappa}(\cdot, y)-\Phi_{\kappa}(\cdot, y)\right) d s \\
& +\frac{1}{(i \sqrt{n} \kappa)^{2}} \nabla \int_{\Gamma} \operatorname{div}_{\Gamma} \mathbf{J}(y) \Phi_{\sqrt{n} \kappa}(\cdot, y) d s \\
& -\frac{1}{(i \kappa)^{2}} \nabla \int_{\Gamma} \operatorname{div}_{\Gamma} \mathbf{J}(y) \Phi_{\kappa}(\cdot, y) d s .
\end{aligned}
$$

Then $\mathbf{U} \in \mathbf{L}^{2}\left(\mathbb{R}^{3}\right)$, curl $\mathbf{U} \in \mathbf{L}^{2}\left(\mathbb{R}^{3}\right)$, $\operatorname{curl}^{2} \mathbf{U} \in \mathbf{L}^{2}\left(\mathbb{R}^{3}\right)$ satisfies (2.21)(2.23); hence, $\mathbf{U}$ defined above is the unique solution to (2.24). Now, for a given $\gamma_{\Gamma} \operatorname{curl} \mathbf{W} \in \mathbf{H}^{1 / 2}(\Gamma)$, let us construct a lifting function $\widetilde{\mathbf{W}} \in \mathbf{H}^{2}\left(\mathbb{R}^{3}\right)[\mathbf{1 6}]$ such that $\gamma_{\Gamma} \operatorname{curl} \widetilde{\mathbf{W}}=\gamma_{\Gamma} \operatorname{curl} \mathbf{W}, \gamma_{\Gamma} \widetilde{\mathbf{W}}=0$ and 
$\|\widetilde{\mathbf{W}}\|_{\mathbf{H}^{2}\left(\mathbb{R}^{3}\right)} \leq c\left\|\gamma_{\Gamma} \operatorname{curl} \widetilde{\mathbf{W}}\right\|_{\mathbf{H}^{\frac{1}{2}(\Gamma)}}$ for some constant $c$. Then

$$
\begin{aligned}
\left|\int_{\Gamma} \gamma_{\Gamma} \operatorname{curl} \mathbf{W} \cdot \mathbf{M} d s\right| & =\left|\int_{\Gamma} \gamma_{\Gamma} \operatorname{curl} \widetilde{\mathbf{W}} \cdot \mathbf{M} d s\right| \\
& =\frac{1}{\left|n \kappa^{2}-\kappa^{2}\right|} \mid \int_{\mathbb{R}^{3} \backslash \Gamma}\left(\operatorname{curl}^{2}+n \kappa^{2}\right) \mathbf{U} \\
& \cdot\left(\operatorname{curl}^{2}+\kappa^{2}\right) \widetilde{\mathbf{W}} d x \mid \\
& \leq\|\mathbf{U}\|_{\mathbf{V}}\|\widetilde{\mathbf{W}}\|_{\mathbf{V}} \leq c\|\mathbf{U}\|_{\mathbf{V}}\left\|\gamma_{\Gamma} \operatorname{curl} \widetilde{\mathbf{W}}\right\|_{\mathbf{H}^{1 / 2}(\Gamma)} .
\end{aligned}
$$

Hence, $\|\mathbf{M}\|_{\mathbf{H}_{t}^{-1 / 2}(\Gamma)} \leq c\|\mathbf{U}\|_{\mathbf{V}}$. Similarly, for given $\gamma_{\Gamma} \mathbf{W} \in \mathbf{H}^{3 / 2}(\Gamma)$, we construct the lifting $\widetilde{\mathbf{W}}_{2} \in \mathbf{H}^{2}\left(\mathbb{R}^{3}\right)[\mathbf{1 6}]$ such that $\gamma_{\Gamma} \widetilde{\mathbf{W}}_{2}=\gamma_{\Gamma} \mathbf{W}$, $\gamma_{\Gamma} \operatorname{curl} \widetilde{\mathbf{W}_{2}}=0$ and $\left\|\widetilde{\mathbf{W}_{2}}\right\|_{\mathbf{H}^{2}\left(\mathbb{R}^{3}\right)} \leq c\left\|\gamma_{T} \widetilde{\mathbf{W}_{2}}\right\|_{\mathbf{H}^{3 / 2}(\Gamma)}$ for some constant $c$. We recall that $\operatorname{div}_{\Gamma} \mathbf{J}=0$; hence, from the Helmoltz decomposition $\mathbf{J}=\overrightarrow{\operatorname{curl}}_{\Gamma} q \in \mathbf{H}^{-3 / 2}(\Gamma)$. Thus, we have

$$
\begin{aligned}
\left|\int_{\Gamma} \gamma_{\Gamma} \mathbf{W} \cdot \mathbf{J} d s\right| & =\left|\int_{\Gamma} \gamma_{\Gamma} \widetilde{\mathbf{W}}_{2} \cdot \mathbf{J} d s\right| \\
& =\frac{1}{\left|n \kappa^{2}-\kappa^{2}\right|}\left|\int_{\mathbb{R}^{3} \backslash \Gamma}\left(\operatorname{curl}^{2}+n \kappa^{2}\right) \mathbf{U} \cdot\left(\operatorname{curl}^{2}+\kappa^{2}\right) \widetilde{\mathbf{W}}_{2} d x\right| \\
& \leq c\|\mathbf{U}\|_{\mathbf{V}}\left\|\widetilde{\mathbf{W}}_{2}\right\|_{\mathbf{V}} \leq c\|\mathbf{U}\|_{\mathbf{V}}\left\|\gamma_{T} \mathbf{W}\right\|_{\mathbf{H}^{3 / 2}(\Gamma)} .
\end{aligned}
$$

Since $\mathbf{J}=\overrightarrow{\operatorname{curl}}_{\Gamma} q \in \mathbf{H}^{-3 / 2}(\Gamma)$, then, by duality,

$$
\|\mathbf{J}\|_{\mathbf{H}_{0}^{-3 / 2,-1 / 2}(\operatorname{div}, \Gamma)} \leq c\|\mathbf{U}\|_{\mathbf{V}}
$$

Finally,

$$
\begin{aligned}
& \left|\left\langle\mathbf{L}(i \kappa)\left(\begin{array}{c}
\mathbf{M} \\
\mathbf{J}
\end{array}\right),\left(\begin{array}{c}
\mathbf{M} \\
\mathbf{J}
\end{array}\right)\right\rangle\right| \\
& =\left\|\int_{\Gamma} \gamma_{\Gamma} \operatorname{curl} \mathbf{U} \cdot \overline{\mathbf{M}} d s+\int_{\Gamma} \gamma_{\Gamma} \mathbf{U} \cdot \overline{\mathbf{J}} d s\right\| \\
& \geq c\|\mathbf{U}\|_{\mathbf{V}} \\
& \geq c\left(\|\mathbf{M}\|_{\mathbf{H}_{t}^{-1 / 2}(\Gamma)}+\|\mathbf{J}\|_{\mathbf{H}^{-3 / 2,-1 / 2}(\operatorname{div}, \Gamma)}\right),
\end{aligned}
$$

where $c$ is a constant depending on $\kappa$. This proves our lemma. 
Next we proceed with the following lemma.

Lemma 2.6. Let

$$
\gamma(k):=\left(k_{1}^{2}-k^{2}\right) /\left(\left|k_{1}\right|^{2}-|k|^{2}\right)
$$

and $k_{1}=k \sqrt{n}$ for $k \in \mathbb{C} \backslash \mathbb{R}_{-}$. Then $\mathbf{L}(k)+\gamma(k) \mathbf{L}(i|k|): \mathbf{H}_{0}(\Gamma) \rightarrow$ $\mathbf{H}^{*}(\Gamma)$ is compact.

Proof. From [15, Theorem 3.8], the operator

$$
\left(\mathbf{S}_{k_{1}}-\mathbf{S}_{k}\right)+\gamma(k)\left(\mathbf{S}_{i\left|k_{1}\right|}-\mathbf{S}_{i|k|}\right): \mathbf{H}^{-3 / 2}(\Gamma) \longrightarrow \mathbf{H}^{3 / 2}(\Gamma)
$$

is compact. Then, from (2.13), we have

$$
\begin{gathered}
\nabla_{\Gamma} \circ\left(S_{k_{1}}-S_{k}\right) \circ \operatorname{div}_{\Gamma}+\gamma(k) \nabla_{\Gamma} \circ\left(S_{i\left|k_{1}\right|}-S_{i|k|}\right) \circ \operatorname{div}_{\Gamma}: \\
\mathbf{H}^{-1 / 2}(\Gamma) \longrightarrow \mathbf{H}^{1 / 2}(\Gamma) \\
\left(\mathbf{K}_{k_{1}}-\mathbf{K}_{k}\right)+\gamma(k)\left(\mathbf{K}_{i\left|k_{1}\right|}-\mathbf{K}_{i|k|}\right): \mathbf{H}^{-3 / 2}(\Gamma) \longrightarrow \mathbf{H}^{1 / 2}(\Gamma) \\
\left(\mathbf{K}_{k_{1}}-\mathbf{K}_{k}\right)+\gamma(k)\left(\mathbf{K}_{i\left|k_{1}\right|}-\mathbf{K}_{i|k|}\right): \mathbf{H}^{-1 / 2}(\Gamma) \longrightarrow \mathbf{H}^{3 / 2}(\Gamma) \\
\left(\frac{1}{k_{1}} \mathbf{K}_{k_{1}}-\frac{1}{k} \mathbf{K}_{k}\right)+\gamma(k)\left(\frac{1}{i\left|k_{1}\right|} \mathbf{K}_{i\left|k_{1}\right|}-\frac{1}{i|k|} \mathbf{K}_{i|k|}\right): \\
\mathbf{H}^{-3 / 2}(\Gamma) \longrightarrow \mathbf{H}^{3 / 2}(\Gamma)
\end{gathered}
$$

are compact. It remains to show that

$$
\begin{aligned}
&\left(k_{1}{ }^{2} \mathbf{S}_{k_{1}}-k^{2} \mathbf{S}_{k}\right)+\gamma(k)\left(\left(i\left|k_{1}\right|\right)^{2} \mathbf{S}_{i\left|k_{1}\right|}-(i|k|)^{2} \mathbf{S}_{i|k|}\right): \\
& \mathbf{H}^{-1 / 2}(\Gamma) \rightarrow \mathbf{H}^{1 / 2}(\Gamma)
\end{aligned}
$$

is compact. Since

$$
\begin{aligned}
& \left(k_{1}^{2} \mathbf{S}_{k_{1}}-k^{2} \mathbf{S}_{k}\right)+\gamma(k)\left(\left(i\left|k_{1}\right|\right)^{2} \mathbf{S}_{i\left|k_{1}\right|}-(i|k|)^{2} \mathbf{S}_{i|k|}\right) \\
& =\left(k_{1}^{2}\left(\mathbf{S}_{k_{1}}-\mathbf{S}_{0}\right)-k^{2}\left(\mathbf{S}_{k}-\mathbf{S}_{0}\right)\right) \\
& \quad+\gamma(k)\left(\left(i\left|k_{1}\right|\right)^{2}\left(\mathbf{S}_{i\left|k_{1}\right|}-\mathbf{S}_{0}\right)-(i|k|)^{2}\left(\mathbf{S}_{i|k|}-\mathbf{S}_{0}\right)\right)
\end{aligned}
$$

and $\mathbf{S}_{k}-\mathbf{S}_{0}$ is compact, then the compactness follows. Hence, the proof of the lemma is completed.

In order to handle the non divergence free part of $\mathbf{J}$, we will split $\mathbf{J}:=\mathbf{Q}+\mathbf{P}$, where $\mathbf{Q} \in \mathbf{H}_{0}^{-3 / 2,-1 / 2}(\operatorname{div}, \Gamma), \mathbf{P}=\nabla_{\Gamma} p \in \mathbf{H}_{t}^{1 / 2}(\Gamma)$ and 
rewrite equation (2.11) for the unknowns $(\mathbf{M}, \mathbf{Q}, \mathbf{P})$. To this end, let us define

$$
\mathbf{H}_{1}(\Gamma):=\left\{\mathbf{P} \in \mathbf{H}_{t}^{1 / 2}(\Gamma), \operatorname{curl}_{\Gamma} \mathbf{P}=0\right\}
$$

and introduce the operator

$$
\widetilde{\mathbf{L}}(k)=\left(\begin{array}{ccc}
k_{1} \mathbf{T}_{k_{1}}-k \mathbf{T}_{k} & \mathbf{K}_{k_{1}}-\mathbf{K}_{k} & \mathbf{K}_{k_{1}}-\mathbf{K}_{k} \\
\mathbf{K}_{k_{1}}-\mathbf{K}_{k} & \mathbf{S}_{k_{1}}-\mathbf{S}_{k} & \mathbf{S}_{k_{1}}-\mathbf{S}_{k} \\
\mathbf{K}_{k_{1}}-\mathbf{K}_{k} & \mathbf{S}_{k_{1}}-\mathbf{S}_{k} & \left(\mathbf{S}_{k_{1}}-\mathbf{S}_{k}\right)+\nabla_{\Gamma} \circ\left(\frac{1}{k_{1}^{2}} \mathbf{S}_{k_{1}}-\frac{1}{k^{2}} \mathbf{S}_{k}\right) \circ \operatorname{div}_{\Gamma}
\end{array}\right) .
$$

From Lemmas 2.1 and 2.2, $\widetilde{\mathbf{L}}(k): \mathbf{H}_{0}(\Gamma) \times \mathbf{H}_{1}(\Gamma) \rightarrow \mathbf{H}^{*}(\Gamma) \times$ $\mathbf{H}^{-1 / 2}(\Gamma)$ is bounded and, furthermore, the family of operators $\widetilde{\mathbf{L}}(k)$ depends analytically on $k \in \mathbb{C} \backslash \mathbb{R}_{-}$, where recall $\mathbf{H}_{0}(\Gamma):=\mathbf{H}_{t}^{-1 / 2}(\Gamma) \times$ $\mathbf{H}_{0}^{-3 / 2,-1 / 2}(\operatorname{div}, \Gamma)$ with its dual $\mathbf{H}^{*}(\Gamma)$. We first notice that $(2.11)$ is equivalent to the following:

$$
\left\langle\mathbf{L}(k)\left(\begin{array}{c}
\mathbf{M} \\
\mathbf{J}
\end{array}\right),\left(\begin{array}{c}
\widetilde{\mathbf{M}} \\
\widetilde{\mathbf{J}}
\end{array}\right)\right\rangle=0
$$

for any $(\widetilde{\mathbf{M}}, \widetilde{\mathbf{J}}) \in \mathbf{H}_{t}^{1 / 2}(\Gamma) \times \mathbf{H}^{-1 / 2,1 / 2}(\operatorname{curl}, \Gamma)$, which equivalently can be written as

$$
\left\langle\widetilde{\mathbf{L}}(k)\left(\begin{array}{c}
\mathbf{M} \\
\mathbf{Q} \\
\mathbf{P}
\end{array}\right),\left(\begin{array}{c}
\widetilde{\mathbf{M}} \\
\widetilde{\mathbf{Q}} \\
\widetilde{\mathbf{P}}
\end{array}\right)\right\rangle=0
$$

for any $(\widetilde{\mathbf{M}}, \widetilde{\mathbf{Q}}, \widetilde{\mathbf{P}}) \in \mathbf{H}^{*} \times \mathbf{H}_{t}^{-1 / 2}(\Gamma)$. Now we are ready to prove the following lemma.

Lemma 2.7. The operator $\widetilde{\mathbf{L}}(k): \mathbf{H}_{0}(\Gamma) \times \mathbf{H}_{1}(\Gamma) \rightarrow \mathbf{H}^{*}(\Gamma) \times \mathbf{H}^{-1 / 2}(\Gamma)$ is Fredholm with index zero, i.e., it can be written as a sum of an invertible operator and a compact operator.

Proof. We rewrite the operator $\widetilde{\mathbf{L}}(k)$ as follows, where $\widetilde{\mathbf{L}}_{1}(k)$ is 


$$
\begin{aligned}
& \widetilde{\mathbf{L}}(k)
\end{aligned}
$$

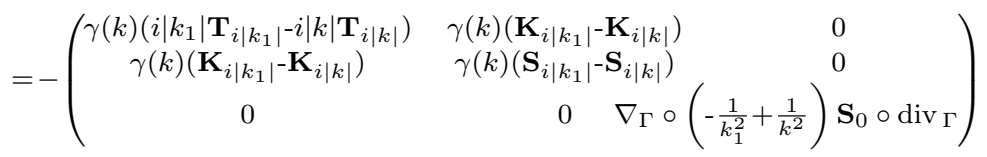

$$
\begin{aligned}
& +\left(\begin{array}{ccc}
\gamma(k)\left(i\left|k_{1}\right| \mathbf{T}_{i\left|k_{1}\right|}-i|k| \mathbf{T}_{i|k|}\right) & \gamma(k)\left(\mathbf{K}_{i\left|k_{1}\right|}-\mathbf{K}_{i|k|}\right) & 0 \\
\gamma(k)\left(\mathbf{K}_{i\left|k_{1}\right|}-\mathbf{K}_{i|k|}\right) & \gamma(k)\left(\mathbf{S}_{i\left|k_{1}\right|}-\mathbf{S}_{i|k|}\right) & 0 \\
0 & 0 & \nabla_{\Gamma} \circ\left(-\frac{1}{k_{1}^{2}}+\frac{1}{k^{2}}\right) \mathbf{S}_{0} \circ \operatorname{div}{ }_{\Gamma}
\end{array}\right) \\
& +\left(\begin{array}{ccc}
k_{1} \mathbf{T}_{k_{1}}-k \mathbf{T}_{k} & \mathbf{K}_{k_{1}}-\mathbf{K}_{k} & \mathbf{K}_{k_{1}}-\mathbf{K}_{k} \\
\mathbf{K}_{k_{1}}-\mathbf{K}_{k} & \mathbf{S}_{k_{1}}-\mathbf{S}_{k} & \mathbf{S}_{k_{1}}-\mathbf{S}_{k} \\
\mathbf{K}_{k_{1}}-\mathbf{K}_{k} & \mathbf{S}_{k_{1}}-\mathbf{S}_{k} & \left(\mathbf{S}_{k_{1}}-\mathbf{S}_{k}\right)+\nabla_{\Gamma} \circ\left(\frac{1}{k_{1}^{2}} \mathbf{S}_{k_{1}}-\frac{1}{k^{2}} \mathbf{S}_{k}\right) \circ \operatorname{div} \Gamma
\end{array}\right) \\
& =: \widetilde{\mathbf{L}}_{1}(k)+\widetilde{\mathbf{L}}_{2}(k)
\end{aligned}
$$

the first operator and $\widetilde{\mathbf{L}}_{2}(k)$ is the sum of the last two operators. Then from Lemma 2.6 and the fact that $\mathbf{S}_{k_{1}}-\mathbf{S}_{k}, \mathbf{K}_{k_{1}}-\mathbf{K}_{k}$ are smoothing operators of orders 3,2 , respectively, we have $\widetilde{\mathbf{L}}_{2}(k)$ is compact. From Lemma 2.5 and the fact that $\mathbf{S}_{0}$ is invertible, whence we have $\widetilde{\mathbf{L}}_{1}(k)$ is invertible. This proves our lemma.

3. The case when $N-I$ changes sign. In this section, we will discuss the Fredholm properties of $\mathbf{L}(k)$ when $N$ is no longer a constant. Our approach for handling the more general case follows exactly the lines of the discussion in [15, Section 4], and here, for the sake of the reader's convenience, we sketch the main steps of the analysis.

3.1. Piecewise homogeneous medium. To begin with, we assume that $D=\bar{D}_{1} \cup \bar{D}_{2}$ such that $D_{1} \subset D$ and $D_{2}:=D \backslash \bar{D}_{1}$ and consider the simple case when $N=n_{2} I$ in $D_{2}$ and $N=n_{1} I$ in $D_{1}$ where $n_{1}>0$ and $n_{2}>0$ are two positive constants such that $\left(n_{1}-1\right)\left(n_{2}-1\right)<0$. Let $\Gamma=\partial D$ and $\Sigma=\partial D_{1}$ which are assumed to be $C^{2}$ smooth surfaces, and let $\nu$ denote the unit normal vector to either $\Gamma$ or $\Sigma$ outward to $D$ and $D_{1}$, respectively (see Figure 1). Let us recall the notation $k_{1}=k \sqrt{n_{1}}$ and $k_{2}=k \sqrt{n_{2}}$. 


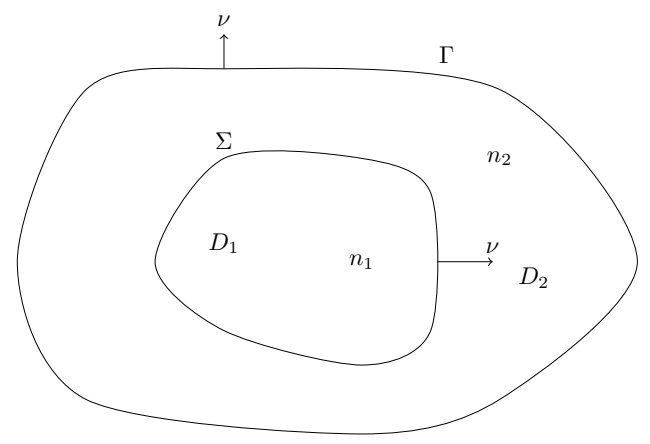

Figure 1. Configuration of the geometry for two constants.

For convenience, we let $\mathbf{K}_{k}^{\Sigma, \Gamma}$ and $\mathbf{T}_{k}^{\Sigma, \Gamma}$ be the potentials $\mathbf{K}_{k}$ and $\mathbf{T}_{k}$ given by (2.5) and (2.6) for densities defined on $\Sigma$ and evaluated on $\Gamma$. The solution of the transmission eigenvalue problem (1.10)-(1.13) by means of the Stratton-Chu formula can be represented as

$$
\begin{aligned}
\mathbf{E}_{0}(x)= & \operatorname{curl} \int_{\Gamma}\left(\mathbf{E}_{0} \times \nu\right)(\mathbf{y}) \Phi_{k}(x, y) d s_{y} \\
& +\int_{\Gamma}\left(\operatorname{curl} \mathbf{E}_{0} \times \nu\right)(\mathbf{y}) \Phi_{k}(\cdot, \mathbf{y}) d s_{y} \\
& +\frac{1}{k^{2}} \nabla \int_{\Gamma} \operatorname{div}_{T}\left(\operatorname{curl} \mathbf{E}_{0} \times \nu\right)(\mathbf{y}) \Phi_{k}(\cdot, \mathbf{y}) d s_{y} \quad \text { in } D
\end{aligned}
$$

$$
\begin{aligned}
\mathbf{E}(x)= & \operatorname{curl} \int_{\Sigma}(\mathbf{E} \times \nu)(\mathbf{y}) \Phi_{k_{1}}(x, y) d s_{y} \\
& +\int_{\Sigma}(\operatorname{curl} \mathbf{E} \times \nu)(\mathbf{y}) \Phi_{k_{1}}(\cdot, \mathbf{y}) d s_{y} \\
& +\frac{1}{k_{1}^{2}} \nabla \int_{\Sigma} \operatorname{div}_{T}(\operatorname{curl} \mathbf{E} \times \nu)(\mathbf{y}) \Phi_{k_{1}}(\cdot, \mathbf{y}) d s_{y} \quad \text { in } D_{1}
\end{aligned}
$$

$$
\begin{aligned}
\mathbf{E}(x)= & \operatorname{curl} \int_{\Gamma}(\mathbf{E} \times \nu)(\mathbf{y}) \Phi_{k_{2}}(x, y) d s_{y} \\
& +\int_{\Gamma}(\operatorname{curl} \mathbf{E} \times \nu)(\mathbf{y}) \Phi_{k_{2}}(\cdot, \mathbf{y}) d s_{y}
\end{aligned}
$$




$$
\begin{aligned}
& +\frac{1}{k_{2}^{2}} \nabla \int_{\Gamma} \operatorname{div}_{T}(\operatorname{curl} \mathbf{E} \times \nu)(\mathbf{y}) \Phi_{k_{2}}(\cdot, \mathbf{y}) d s_{y} \\
& -\operatorname{curl} \int_{\Sigma}(\mathbf{E} \times \nu)(\mathbf{y}) \Phi_{k_{2}}(x, y) d s_{y} \\
& -\int_{\Sigma}(\operatorname{curl} \mathbf{E} \times \nu)(\mathbf{y}) \Phi_{k_{2}}(\cdot, \mathbf{y}) d s_{y} \\
& -\frac{1}{k_{2}^{2}} \nabla \int_{\Sigma} \operatorname{div}_{T}(\operatorname{curl} \mathbf{E} \times \nu)(\mathbf{y}) \Phi_{k_{2}}(\cdot, \mathbf{y}) d s_{y} \quad \text { in } D_{2} .
\end{aligned}
$$

Let $\mathbf{E} \times \nu=\mathbf{E}_{0} \times \nu=\mathbf{M}, \operatorname{curl} \mathbf{E} \times \nu=\operatorname{curl} \mathbf{E}_{0} \times \nu=\mathbf{J}$ on $\Gamma$ and $\mathbf{E} \times \nu=\mathbf{M}^{\prime}, \operatorname{curl} \mathbf{E} \times \nu=\mathbf{J}^{\prime}$ on $\Sigma$. From the jump relations of the boundary integral operators across $\Gamma$ and $\Sigma$ we have that

$$
\begin{gathered}
\left(\begin{array}{cc}
k_{2} \mathbf{T}_{k_{2}}^{\Gamma}-k \mathbf{T}_{k}^{\Gamma} & \mathbf{K}_{k_{2}}^{\Gamma}-\mathbf{K}_{k}^{\Gamma} \\
\mathbf{K}_{k_{2}}^{\Gamma}-\mathbf{K}_{k}^{\Gamma} & \frac{1}{k_{2}} \mathbf{T}_{k_{2}}^{\Gamma}-\frac{1}{k} \mathbf{T}_{k}^{\Gamma}
\end{array}\right)\left(\begin{array}{c}
\mathbf{M} \\
\mathbf{J}
\end{array}\right) \\
=\left(\begin{array}{cc}
k_{2} \mathbf{T}_{k_{2}}^{\Sigma, \Gamma} & \mathbf{K}_{k_{2}}^{\Sigma, \Gamma} \\
\mathbf{K}_{k_{2}}^{\Sigma, \Gamma} & \frac{1}{k_{2}} \mathbf{T}_{k_{2}}^{\Sigma, \Gamma}
\end{array}\right)\left(\begin{array}{c}
\mathbf{M}^{\prime} \\
\mathbf{J}^{\prime}
\end{array}\right) \\
\left(\begin{array}{c}
k_{2} \mathbf{T}_{k_{2}}^{\Sigma}+k_{1} \mathbf{T}_{k_{1}}^{\Sigma} \\
\mathbf{K}_{k_{2}}^{\Sigma}+\mathbf{K}_{k_{1}}^{\Sigma} \\
\mathbf{K}_{k_{2}}^{\Sigma}+\mathbf{K}_{k_{1}}^{\Sigma} \\
\frac{1}{k_{2}} \mathbf{T}_{k_{2}}^{\Sigma}+\frac{1}{k_{1}} \mathbf{T}_{k_{1}}^{\Sigma}
\end{array}\right)\left(\begin{array}{c}
\mathbf{M}^{\prime} \\
\mathbf{J}^{\prime}
\end{array}\right) \\
=\left(\begin{array}{cc}
k_{2} \mathbf{T}_{k_{2}}^{\Gamma, \Sigma} & \mathbf{K}_{k_{2}}^{\Gamma, \Sigma} \\
\mathbf{K}_{k_{2}}^{\Gamma, \Sigma} & \frac{1}{k_{2}} \mathbf{T}_{k_{2}}^{\Gamma, \Sigma}
\end{array}\right)\left(\begin{array}{c}
\mathbf{M} \\
\mathbf{J}
\end{array}\right) .
\end{gathered}
$$

Let us denote by $\mathbf{L}_{20}(k), \mathbf{L}^{\Sigma, \Gamma}(k), \mathbf{L}_{21}(k)$ and $\mathbf{L}^{\Gamma, \Sigma}(k)$ the matrixvalued operators in the above two equations in order from left to right and from top to bottom, respectively. By the regularity of the solution of the Maxwell's equations inside $D_{2}$ (see e.g. [20]), we have $\left(\mathbf{M}^{\prime}, \mathbf{J}^{\prime}\right) \in \mathbf{H}_{t}^{-1 / 2}(\Sigma, \operatorname{div}) \times \mathbf{H}_{t}^{-1 / 2}(\Sigma, \operatorname{div})$. Then the equation

$$
\mathbf{L}_{21}(k)\left(\begin{array}{c}
\mathbf{M}^{\prime} \\
\mathbf{J}^{\prime}
\end{array}\right)=\left(\begin{array}{c}
\mathbf{g} \\
\mathbf{h}
\end{array}\right)
$$

where $(\mathbf{g}, \mathbf{h}) \in \mathbf{H}_{t}^{-1 / 2}(\Sigma$, div $) \times \mathbf{H}_{t}^{-1 / 2}(\Sigma$, div $)$ corresponds to the transmission problem which is to find $\left(\mathbf{E}_{2}, \mathbf{E}_{1}\right) \in \mathbf{H}_{\text {loc }}\left(\operatorname{curl}, \mathbb{R}^{3} \backslash \overline{D_{1}}\right) \times$ 
$\mathbf{H}\left(\operatorname{curl}, D_{1}\right)$ and $\mathbf{E}_{2}$ such that

$$
\begin{aligned}
\text { curl curl } \mathbf{E}_{2}-k_{2}^{2} \mathbf{E}_{2}=0 & \text { in } \mathbb{R}^{3} \backslash \overline{D_{1}} \\
\text { curl curl } \mathbf{E}_{1}-k_{1}^{2} \mathbf{E}_{1}=0 & \text { in } D_{1} \\
\nu \times \mathbf{E}_{2}-\nu \times \mathbf{E}_{1}=\mathbf{g} & \text { on } \Sigma \\
\nu \times\left(\operatorname{curl} \mathbf{E}_{2}\right)-\nu \times\left(\operatorname{curl} \mathbf{E}_{1}\right)=\mathbf{h} & \text { on } \Sigma,
\end{aligned}
$$

and $\mathbf{E}_{2}$ satisfies the Silver-Mueller radiation condition. By wellposedness of the transmission problem we have $\mathbf{L}_{21}(k)$ is invertible. Hence, plugging in (3.4) $\mathbf{M}^{\prime}$ and $\mathbf{J}^{\prime}$ from (3.5), we obtain the following equation for $\mathbf{M}$ and $\mathbf{J}$

$$
\mathbf{L}(k)\left(\begin{array}{c}
\mathbf{M} \\
\mathbf{J}
\end{array}\right)=\left(\begin{array}{l}
0 \\
0
\end{array}\right)
$$

where $\mathbf{L}(k):=\mathbf{L}_{20}(k)-\mathbf{L}^{\Sigma, \Gamma}(k) \mathbf{L}_{21}(k)^{-1} \mathbf{L}^{\Gamma, \Sigma}(k)$. Then in a similar way to Theorem 2.4, we can prove the following theorem.

Theorem 3.1. The following statements are equivalent:

(i) There exist non trivial $\mathbf{E}, \mathbf{E}_{0} \in L^{2}(D), \mathbf{E}-\mathbf{E}_{0} \in \mathbf{H}\left(\operatorname{curl}^{2}, D\right)$, such that (2.1)-(2.4) holds.

(ii) There exists non trivial $(\mathbf{M}, \mathbf{J}) \in \mathbf{H}_{t}^{-1 / 2}(\Gamma) \times \mathbf{H}^{-3 / 2,-1 / 2}(\operatorname{div}, \Gamma)$ such that (3.6) holds and $\mathbf{E}_{0}^{\infty}(\mathbf{M}, \mathbf{J})=0$, where

$$
\begin{aligned}
\mathbf{E}_{0}^{\infty}(\mathbf{M}, \mathbf{J})(\widehat{x})= & \widehat{x} \\
\times & \left(\frac{1}{4 \pi} \operatorname{curl} \int_{\Gamma} \mathbf{M}(y) e^{-i k \hat{x} \cdot y} d s_{y}\right. \\
& \left.+\frac{1}{4 \pi k^{2}} \nabla \int_{\Gamma} \operatorname{div}_{\Gamma} \mathbf{J}(y) e^{-i k \hat{x} \cdot y} d s_{y}+\int_{\Gamma} \mathbf{J}(y) e^{-i k \hat{x} \cdot y} d s_{y}\right) \\
& \times \widehat{x} .
\end{aligned}
$$

Now we note that $\Sigma$ and $\Gamma$ are two disjoint curves and, hence, we have that $\mathbf{L}^{\Sigma, \Gamma}(k), \mathbf{L}^{\Gamma, \Sigma}(k)$ are compact. By writing $\mathbf{L}(k)$ as a $3 \times 3$ matrix operator $\widetilde{\mathbf{L}}(k)$ similar to (2.26), we can have the following lemma directly from Lemma 2.7 .

Lemma 3.2. The operator $\widetilde{\mathbf{L}}(k): \mathbf{H}_{0}(\Gamma) \times \mathbf{H}_{1}(\Gamma) \rightarrow \mathbf{H}^{*}(\Gamma) \times \mathbf{H}^{-1 / 2}(\Gamma)$ is Fredholm with index zero, i.e., it can be written as a sum of an 
invertible operator and a compact operator. Furthermore, the family of the operators $\widetilde{\mathbf{L}}(k)$ depends analytically on $k \in \mathbb{C} \backslash \mathbb{R}_{-}$.

This approach can be readily generalized to the case when the medium consists of finitely many homogeneous layers.

3.2. General inhomogeneous medium. In a more general case where $N=n(x) I$ in $D_{1}$, where $n \in L^{\infty}\left(D_{1}\right)$ such that $n(x) \geq$ $\alpha>0$ but still constant in $D_{2}$, we can prove the same result as in Lemma 3.2 by replacing fundamental solution $\Phi_{k_{1}}(\cdot, y)$ with the free space fundamental $\mathbb{G}(\cdot, y)$ of

$$
\Delta \mathbb{G}(\cdot, y)+k^{2} n(x) \mathbb{G}(\cdot, y)=-\delta_{y} \quad \text { in } \mathbb{R}^{3}
$$

in the distributional sense together with the Sommerfeld radiation condition, where $n(x)$ is extended by its constant value in $D_{2}$ to the whole space $\mathbb{R}^{3}$. Because $\Phi_{k_{2}}(\cdot, y)-\mathbb{G}(\cdot, y)$ solves the Helmholtz equation with wave number $k_{2}$ in the neighborhood of $\Gamma$, the mapping properties of the integral operators do not change. We refer the reader to [15, subsection 4.2] for more details.

In fact, the above idea can be applied even in a more general case, provided that $N$ is a positive constant not equal to one in a neighborhood of $\Gamma$. More precisely, consider a neighborhood $\mathcal{O}$ of $\Gamma$ in $D$ (above denoted by $D_{2}$ ) with $C^{2}$ smooth boundary (e.g., one can take $\mathcal{O}$ to be the region in $D$ bounded by $\Gamma$ and $\Sigma:=\{x-\epsilon \nu(x), x \in \Gamma\}$ for some $\epsilon>0$ where $\nu$ is the outward unit normal vector to $\Gamma$ ). Assume that $N=n I$ in $\mathcal{O}$, where $n \neq 1$ is a positive constant, whereas in $D \backslash \overline{\mathcal{O}}, N$ satisfies the assumptions at the beginning of the paper, i.e., $N$ is a $3 \times 3$ matrix-valued function with $L^{\infty}(D)$ entries such that $\bar{\xi} \cdot \operatorname{Re}(N) \xi \geq \alpha>0$ and $\bar{\xi} \cdot \operatorname{Im}(N) \xi \geq 0$ for every $\xi \in \mathbb{C}^{3}$. Then a similar result as in Theorem 3.1 and Lemma 3.2 holds true in this case. Indeed, without going into details, we can express $\mathbf{E}_{0}$ by (3.1) and $\mathbf{E}$ by (3.3) in $\mathcal{O}$ and in $D \backslash \overline{\mathcal{O}}$ we can leave it in the form of a partial differential equation with Cauchy data connected to $\mathbf{E}$ in $\mathcal{O}$. Hence, it is possible to obtain an equation of the form (3.6) where the operator $\mathbf{L}(k)$ is written as

$$
\mathbf{L}(k)=\mathbf{L}_{n}(k)-\mathbf{L}^{\Sigma, \Gamma}(k) \mathbf{A}^{-1}(k) \mathbf{L}^{\Gamma, \Sigma}(k),
$$


where $\mathbf{L}_{n}(k)$ is the boundary integral operator corresponding to the transmission eigenvalue problem with contrast $n-1$, the compact operators $\mathbf{L}^{\Sigma, \Gamma}(k)$ and $\mathbf{L}^{\Gamma, \Sigma}(k)$ are defined right below (3.4) and (3.5) and $\mathbf{A}(k)$ is the invertible solution operator corresponding to the wellposed transmission problem

$$
\begin{aligned}
\text { curl curl } \mathbf{E}_{2}-k^{2} n_{2} \mathbf{E}_{2}=0 & \text { in } \mathbb{R}^{3} \backslash \overline{D_{1}} \\
\text { curl curl } \mathbf{E}_{1}-k^{2} N \mathbf{E}_{1}=0 & \text { in } D_{1} \\
\nu \times \mathbf{E}_{2}-\nu \times \mathbf{E}_{1}=\mathbf{g} & \text { on } \Sigma \\
\nu \times\left(\operatorname{curl} \mathbf{E}_{2}\right)-\nu \times\left(\operatorname{curl} \mathbf{E}_{1}\right)=\mathbf{h} & \text { on } \Sigma
\end{aligned}
$$

and $\mathbf{E}_{2}$ satisfies the Silver-Müller radiation condition. Hence, the above analysis can apply to prove analogues Theorem 3.1 and Lemma 3.2.

For later use in the following we formally state the assumptions on $N$ (here $\mathcal{O}$ is a neighborhood of $\Gamma$ as explained above).

Assumption 3.2.1. $N$ is a $3 \times 3$ symmetric matrix-valued function with $L^{\infty}(D)$ entries such that $\bar{\xi} \cdot \operatorname{Re}(N) \xi \geq \alpha>0$ and $\bar{\xi} \cdot \operatorname{Im}(N) \xi \geq 0$ for every $\xi \in \mathbb{C}^{3},|\xi|=1$ and $N=n I$ in $\mathcal{O}$ where $n \neq 1$ is a positive constant.

4. The existence of non transmission eigenvalue wave numbers. In this section, we assume that $N$ satisfies Assumption 3.2.1, consider pure imaginary wave numbers $k$ and, for convenience, let $\lambda:=-k^{2}$ be a real positive number in order to start by proving an a priori estimate following the idea of [29] for the scalar case.

Lemma 4.1. Assume that $N$ satisfies Assumption 3.2.1, $\chi(x) \in$ $\mathbb{C}_{0}^{\infty}(D)$ is a real valued cutoff function with $0 \leq \chi \leq 1$ and $\chi \equiv 1$ in $D \backslash \overline{\mathcal{O}}$. If $\mathbf{v} \in \mathbf{L}^{2}(D)$ and

$$
(\operatorname{curl} \operatorname{curl}+\lambda) \mathbf{v}=0 \quad \text { in } D,
$$

then there exists a constant $K(\chi)$ such that, for sufficiently large $\lambda$,

$$
\|\chi \mathbf{v}\|^{2} \leq K \frac{\|(1-\chi) \mathbf{v}\|^{2}}{\lambda} .
$$


Proof. Since $\chi \in \mathbb{C}_{0}^{\infty}(D)$, we have

$$
\begin{aligned}
0= & \int_{D}(\operatorname{curl} \operatorname{curl}+\lambda) \mathbf{v} \cdot\left(\chi^{2} \overline{\mathbf{v}}\right) d x \\
= & \int_{D} \operatorname{curl} \operatorname{curl} \mathbf{v} \cdot\left(\chi^{2} \overline{\mathbf{v}}\right) d x+\lambda \int_{D} \mathbf{v} \cdot\left(\chi^{2} \overline{\mathbf{v}}\right) d x \\
= & \int_{D} \operatorname{curl} \mathbf{v} \cdot \operatorname{curl}\left(\chi^{2} \overline{\mathbf{v}}\right) d x+\lambda \int_{D} \mathbf{v} \cdot\left(\chi^{2} \overline{\mathbf{v}}\right) d x \\
= & \int_{D} \operatorname{curl} \mathbf{v} \cdot(\chi \operatorname{curl}(\chi \overline{\mathbf{v}})) d x \\
& +\int_{D} \operatorname{curl} \mathbf{v} \cdot(\nabla \chi \times(\chi \overline{\mathbf{v}})) d x+\lambda \int_{D} \mathbf{v} \cdot\left(\chi^{2} \overline{\mathbf{v}}\right) d x \\
= & \int_{D} \operatorname{curl}(\chi \mathbf{v}) \cdot \operatorname{curl}(\chi \overline{\mathbf{v}}) d x \\
& -\int_{D} \operatorname{curl}(\chi \overline{\mathbf{v}}) \cdot(\nabla \chi \times \mathbf{v}) d x \\
& +\int_{D} \operatorname{curl} \mathbf{v} \cdot(\nabla \chi \times(\chi \overline{\mathbf{v}})) d x \\
& +\lambda \int_{D} \mathbf{v} \cdot\left(\chi^{2} \overline{\mathbf{v}}\right) d x \\
= & \int_{D}|\operatorname{curl}(\chi \mathbf{v})|^{2} d x-\int_{D}(\chi \operatorname{curl} \overline{\mathbf{v}}+\nabla \chi \times \overline{\mathbf{v}}) \cdot(\nabla \chi \times \mathbf{v}) d x \\
& +\int_{D} \operatorname{curl} \mathbf{v} \cdot(\nabla \chi \times(\chi \overline{\mathbf{v}})) d x+\lambda \int_{D} \mathbf{v} \cdot\left(\chi^{2} \overline{\mathbf{v}}\right) d x \\
= & \int_{D}|\operatorname{curl}(\chi \mathbf{v})|^{2} d x-\int_{D}|(\nabla \chi \times \mathbf{v})|^{2} d x+\lambda \int_{D}|\chi \mathbf{v}|^{2} d x \\
& +\int_{D}((\chi \operatorname{curl} \mathbf{v}) \cdot(\nabla \chi \times \overline{\mathbf{v}})-(\chi \operatorname{curl} \overline{\mathbf{v}}) \cdot(\nabla \chi \times \mathbf{v})) d x
\end{aligned}
$$

Taking the real part yields

$$
\int_{D}|\operatorname{curl}(\chi \mathbf{v})|^{2} d x+\lambda \int_{D}|\chi \mathbf{v}|^{2} d x=\int_{D}|(\nabla \chi \times \mathbf{v})|^{2} d x
$$

and then

$$
\lambda\|\chi \mathbf{v}\|^{2} \leq K(\chi)\|\mathbf{v}\|^{2} \leq K(\chi)\left(\|\chi \mathbf{v}\|^{2}+\|(1-\chi) \mathbf{v}\|^{2}\right),
$$

which yields (4.1) for sufficiently large $\lambda$. 
Now we are ready to prove the following theorem.

Theorem 4.2. Under Assumption 3.2.1, there exists a sufficiently large real $\lambda>0$ where $\lambda=-k^{2}$ such that (1.10)-(1.13) has only trivial solutions.

Proof. Assume first $n-1<0$ in $\mathcal{O}$, let $\mathbf{u}=\mathbf{E}-\mathbf{E}_{0} \in \mathbf{H}_{0}\left(\operatorname{curl}^{2}, D\right)$ and $\mathbf{v}=\lambda \mathbf{E}_{0} \in \mathbf{L}^{2}(D)$, then

$$
\begin{array}{rr}
\operatorname{curl} \operatorname{curl} \mathbf{u}+\lambda N \mathbf{u}=-(N-I) \mathbf{v} & \text { in } D \\
\text { curlcurl } \mathbf{v}+\lambda \mathbf{v}=0 & \text { in } D \\
\nu \times \mathbf{u}=\nu \times(\operatorname{curl} \mathbf{u})=0 & \text { on } \Gamma .
\end{array}
$$

Then, for any $\varphi \in \mathbf{C}_{0}^{\infty}(D)$, interpreting (4.3) in the distributional sense yields

$$
\int_{D} \mathbf{v}(\operatorname{curl} \operatorname{curl} \varphi+\lambda \varphi)=0
$$

and hence the denseness of $\mathbf{C}_{0}^{\infty}(D)$ in $\mathbf{H}_{0}\left(\operatorname{curl}^{2}, D\right)$ (see [16]) yields

$$
\int_{D} \overline{\mathbf{v}} \cdot \operatorname{curl}^{2} \mathbf{u}+\lambda \int_{D} \overline{\mathbf{v}} \cdot \mathbf{u}=0
$$

Multiplying (4.2) by $\overline{\mathbf{v}}$ yields

$$
\int_{D} \overline{\mathbf{v}} \cdot \operatorname{curl}^{2} \mathbf{u} d x+\lambda \int_{D} N \mathbf{u} \cdot \overline{\mathbf{v}} d x+\int_{D}(N-I) \mathbf{v} \cdot \overline{\mathbf{v}} d x=0
$$

Combining the above with (4.5) yields

$$
\lambda \int_{D}(N-I) \overline{\mathbf{u}} \cdot \mathbf{v} d x+\int_{D}(N-I) \mathbf{v} \cdot \overline{\mathbf{v}} d x=0
$$

Multiplying (4.2) by $\overline{\mathbf{u}}$ and integrating by parts yields

$$
\int_{D}|\operatorname{curl} \mathbf{u}|^{2} d x+\lambda \int_{D} N \mathbf{u} \cdot \overline{\mathbf{u}} d x+\int_{D}(N-I) \mathbf{v} \cdot \overline{\mathbf{u}} d x=0
$$

Noting that $N$ is symmetric, we have $(N-I) \overline{\mathbf{u}} \cdot \mathbf{v}=(N-I) \mathbf{v} \cdot \overline{\mathbf{u}}$, and hence

$$
\int_{D}|\operatorname{curl} \mathbf{u}|^{2} d x+\lambda \int_{D} N \mathbf{u} \cdot \overline{\mathbf{u}} d x+\int_{D}(N-I) \overline{\mathbf{u}} \cdot \mathbf{v} d x=0
$$


By regularity [26] $\mathbf{v}$ is sufficiently smooth in $D$ away from the boundary and hence by unique continuation we can see $\int_{\mathcal{O}}(n-1)\left(1-\chi^{2}\right)|\mathbf{v}|^{2} d x \neq$ 0 . Then combining (4.6) with (4.7) yields

$$
\text { (4.8) } \begin{aligned}
& \int_{D}|\operatorname{curl} \mathbf{u}|^{2} d x+\lambda \int_{D} N \mathbf{u} \cdot \overline{\mathbf{u}} d x=\frac{1}{\lambda} \int_{D}(N-I) \mathbf{v} \cdot \overline{\mathbf{v}} d x \\
= & \frac{1}{\lambda}\left(\int_{D}(N-I) \chi^{2} \mathbf{v} \cdot \overline{\mathbf{v}} d x+\int_{D}(N-I)\left(1-\chi^{2}\right) \mathbf{v} \cdot \overline{\mathbf{v}} d x\right) \\
(4.9)= & \frac{1}{\lambda} \int_{D}(N-I)\left(1-\chi^{2}\right) \mathbf{v} \cdot \overline{\mathbf{v}} d x\left(1+\frac{\int_{D}(N-I) \chi^{2} \mathbf{v} \cdot \overline{\mathbf{v}} d x}{\int_{D}(N-I)\left(1-\chi^{2}\right) \mathbf{v} \cdot \overline{\mathbf{v}} d x}\right) \\
= & \frac{1}{\lambda}(n-1) \int_{\mathcal{O}}\left(1-\chi^{2}\right)|\mathbf{v}|^{2} d x\left(1+\frac{\int_{D}(N-I) \chi^{2} \mathbf{v} \cdot \overline{\mathbf{v}} d x}{(n-1) \int_{\mathcal{O}}\left(1-\chi^{2}\right)|\mathbf{v}|^{2} d x}\right)
\end{aligned}
$$

From Lemma 4.1, we have for sufficiently large $\lambda$,

$$
\frac{\left|\int_{D}(N-I) \chi^{2} \mathbf{v} \cdot \overline{\mathbf{v}} d x\right|}{(1-n) \int_{\mathcal{O}}\left(1-\chi^{2}\right)|\mathbf{v}|^{2} d x}<\frac{K\left(N_{\max }+1\right)}{\lambda}<1
$$

where $N_{\max }$ is supremum over $D$ of the largest eigenvalue of $N$, which implies

$$
\Re\left(1+\frac{\int_{D}(N-I) \chi^{2} \mathbf{v} \cdot \overline{\mathbf{v}} d x}{(n-1) \int_{\mathcal{O}}\left(1-\chi^{2}\right)|\mathbf{v}|^{2} d x}\right)>0 .
$$

Then, since $n-1<0$, the real part of $(4.9)$ is non positive for sufficiently large $\lambda$ but the real part of (4.8) is non negative. Hence, the only possibility is $\mathbf{u}=0, \mathbf{v}=0$, i.e., $\mathbf{E}=\mathbf{E}_{0}=0$.

Let us next consider $n-1>0$ in $\mathcal{O}$, and let $\mathbf{u}=\mathbf{E}-\mathbf{E}_{0}, \mathbf{v}=\lambda \mathbf{E}$. Then

$$
\begin{aligned}
\text { curl curl } \mathbf{u}+\lambda \mathbf{u}=-(N-I) \mathbf{v} & \text { in } D \\
\operatorname{curl} \operatorname{curl} \mathbf{v}+\lambda N \mathbf{v}=0 & \text { in } D \\
\nu \times \mathbf{u}=\nu \times(\operatorname{curl} \mathbf{u})=0 & \text { on } \Gamma
\end{aligned}
$$

Using the same argument as for (4.5),

$$
\int_{D} \operatorname{curl}^{2} \overline{\mathbf{u}} \cdot \mathbf{v} d x+\lambda \int_{D} N \mathbf{v} \cdot \overline{\mathbf{u}} d x=0
$$

Multiplying (4.10) by $\overline{\mathbf{v}}$ yields

$$
\int_{D} \overline{\mathbf{v}} \cdot \operatorname{curl}^{2} \mathbf{u} d x+\lambda \int_{D} \overline{\mathbf{v}} \cdot \mathbf{u} d x+\int_{D}(N-I) \mathbf{v} \cdot \overline{\mathbf{v}} d x=0
$$


Combining the conjugate of the above with (4.13) yields

$$
\lambda \int_{D}(N-I) \overline{\mathbf{u}} \cdot \mathbf{v} d x=\int_{D} \overline{N-I} \overline{\mathbf{v}} \cdot \mathbf{v} d x
$$

Multiplying (4.10) by $\overline{\mathbf{u}}$ and integrating by parts yields

$$
\int_{D}|\operatorname{curl} \mathbf{u}|^{2} d x+\lambda \int_{D}|\mathbf{u}|^{2} d x+\int_{D}(N-I) \mathbf{v} \cdot \overline{\mathbf{u}} d x=0 .
$$

Note that, since $N$ is symmetric, then $(N-I) \overline{\mathbf{u}} \cdot \mathbf{v}=(N-I) \mathbf{v} \cdot \overline{\mathbf{u}}$, and hence

$$
\int_{D}|\operatorname{curl} \mathbf{u}|^{2} d x+\lambda \int_{D}|\mathbf{u}|^{2} d x+\int_{D}(N-I) \overline{\mathbf{u}} \cdot \mathbf{v} d x=0 .
$$

Then, combining (4.14) with (4.15) yields

$$
\begin{aligned}
& \int_{D}|\operatorname{curl} \mathbf{u}|^{2} d x+\lambda \int_{D}|\mathbf{u}|^{2} d x \\
& =-\frac{1}{\lambda} \int_{D} \overline{N-I} \overline{\mathbf{v}} \cdot \mathbf{v} d x \\
& =-\frac{1}{\lambda}\left(\int_{D} \chi^{2} \overline{N-I} \overline{\mathbf{v}} \cdot \mathbf{v} d x+\int_{D}\left(1-\chi^{2}\right) \overline{N-I} \overline{\mathbf{v}} \cdot \mathbf{v} d x\right) \\
& =-\frac{1}{\lambda} \int_{D}\left(1-\chi^{2}\right) \overline{N-I} \overline{\mathbf{v}} \cdot \mathbf{v} d x\left(1+\frac{\int_{D} \chi^{2} \overline{N-I} \overline{\mathbf{v}} \cdot \mathbf{v} d x}{\int_{D}\left(1-\chi^{2}\right) \overline{N-I} \overline{\mathbf{v}} \cdot \mathbf{v} d x}\right) \\
& =-\frac{1}{\lambda} \int_{\mathcal{O}}(n-1)\left(1-\chi^{2}\right)|\mathbf{v}|^{2} d x\left(1+\frac{\int_{D} \chi^{2} \overline{N-I} \overline{\mathbf{v}} \cdot \mathbf{v} d x}{(n-1) \int_{\mathcal{O}}\left(1-\chi^{2}\right)|\mathbf{v}|^{2} d x}\right) .
\end{aligned}
$$

From Lemma 4.1, we have, for sufficiently large $\lambda$,

$$
\frac{\left|\int_{D} \chi^{2} \overline{N-I} \overline{\mathbf{v}} \cdot \mathbf{v} d x\right|}{(n-1) \int_{\mathcal{O}}\left(1-\chi^{2}\right)|\mathbf{v}|^{2} d x}<\frac{K\left(N_{\max }+1\right)}{\lambda}<1 .
$$

Then

$$
\Re\left(1+\frac{\int_{D} \chi^{2} \overline{N-I} \overline{\mathbf{v}} \cdot \mathbf{v} d x}{\int_{\mathcal{O}}(n-1)\left(1-\chi^{2}\right)|\mathbf{v}|^{2} d x}\right)>0 .
$$

Therefore, since $n-1>0$, the real part of (4.16) is non positive for sufficiently large $\lambda$, but the real part of the righthand side of (4.16) is non positive for sufficiently large $\lambda$, and hence the real part of the 
lefthand side of (4.16) is non negative. Hence, the only possibility is $\mathbf{u}=0, \mathbf{v}=0$, i.e., $\mathbf{E}=\mathbf{E}_{0}=0$.

5. Discreteness of transmission eigenvalues. Recall that in Section 3, we have proved that $\widetilde{\mathbf{L}}(k)$ is a Fredholm operator. Hence, to show discreteness, we will use the analytic Fredholm theory [11]. To this end, we must show that there exists $k$ such that $\widetilde{\mathbf{L}}(k)$ is injective.

Lemma 5.1. Assume that $N$ satisfies Assumption 3.2.1. There exists a purely imaginary $k$ with sufficiently large $|k|>0$ such that $\widetilde{\mathbf{L}}(k)$ is injective.

Proof. Let us extend $N$ to $\mathbb{R}^{3} \backslash \bar{D}$ by $N=n I$ where $n$ is the constant $\left.N\right|_{\mathcal{O}}$. Assume there exists $\left(\begin{array}{c}\mathbf{M} \\ \mathbf{J}\end{array}\right)$ such that $\mathbf{L}(k)\left(\begin{array}{c}\mathbf{M} \\ \mathbf{J}\end{array}\right)=0$. We will show that if $k$ is purely imaginary with large modulus, then $\left(\begin{array}{c}\mathbf{M} \\ \mathbf{J}\end{array}\right)=0$. Recalling (3.7), we define

$$
\left(\begin{array}{c}
\mathbf{M}^{\prime} \\
\mathbf{J}^{\prime}
\end{array}\right)=\mathcal{A}^{-1}(k) \mathbf{L}^{\Gamma, \Sigma}(k)\left(\begin{array}{c}
\mathbf{M} \\
\mathbf{J}
\end{array}\right)
$$

and

$$
\begin{aligned}
\mathbf{E}_{0}(x)= & \operatorname{curl} \int_{\Gamma} \mathbf{M}(\mathbf{y}) \Phi_{k}(x, y) d s_{y} \\
& +\int_{\Gamma} \mathbf{J}(\mathbf{y}) \Phi_{k}(\cdot, \mathbf{y}) d s_{y} \\
& +\frac{1}{k^{2}} \nabla \int_{\Gamma} \operatorname{div}_{T} \mathbf{J}(\mathbf{y}) \Phi_{k}(\cdot, \mathbf{y}) d s_{y} \quad \text { in } \mathbb{R}^{3} \backslash \Gamma .
\end{aligned}
$$

From the definition of $\left(\begin{array}{c}\mathbf{M}^{\prime} \\ \mathbf{J}^{\prime}\end{array}\right)$, there exist $\mathbf{E} \in \mathbf{L}\left(D_{1}\right)$ and $D_{1}:=$ $D \backslash \overline{\mathcal{O}}$, such that

$$
\begin{aligned}
\text { curl curl } \mathbf{E}-k^{2} N \mathbf{E}=0 & \text { in } D_{1} \\
{[\mathbf{E} \times \nu]^{+}=\mathbf{M}^{\prime} } & \text { on } \Sigma \\
{[\operatorname{curl} \mathbf{E} \times \nu]^{+}=\mathbf{J}^{\prime} } & \text { on } \Sigma .
\end{aligned}
$$


Also, we define

$$
\begin{aligned}
\mathbf{E}(x)= & \operatorname{curl} \int_{\Gamma} \mathbf{M}(\mathbf{y}) \Phi_{k_{2}}(x, y) d s_{y} \\
& +\int_{\Gamma} \mathbf{J}(\mathbf{y}) \Phi_{k_{2}}(\cdot, \mathbf{y}) d s_{y} \\
& +\frac{1}{k_{2}^{2}} \nabla \int_{\Gamma} \operatorname{div}{ }_{T} \mathbf{J}(\mathbf{y}) \Phi_{k_{2}}(\cdot, \mathbf{y}) d s_{y} \\
& -\operatorname{curl} \int_{\Sigma} \mathbf{M}^{\prime}(\mathbf{y}) \Phi_{k_{2}}(x, y) d s_{y} \\
& -\int_{\Sigma} \mathbf{J}^{\prime}(\mathbf{y}) \Phi_{k_{2}}(\cdot, \mathbf{y}) d s_{y} \\
& -\frac{1}{k_{2}^{2}} \nabla \int_{\Sigma} \operatorname{div}_{T} \mathbf{J}^{\prime}(\mathbf{y}) \Phi_{k_{2}}(\cdot, \mathbf{y}) d s_{y} \quad \text { in } \mathbb{R}^{3} \backslash\left(\bar{D}_{1} \cup \Gamma\right) .
\end{aligned}
$$

Jump relations across $\Gamma$ applied to $\mathbf{E}$ and $\mathbf{E}_{0}$ along with equation (3.6) yield

$$
\begin{aligned}
\text { curl curl } \mathbf{E}-k^{2} N \mathbf{E}=0 & \text { in } \mathbb{R}^{3} \backslash \Gamma \\
\text { curl curl } \mathbf{E}_{0}-k^{2} \mathbf{E}_{0}=0 & \text { in } \mathbb{R}^{3} \backslash \Gamma \\
(\nu \times \mathbf{E})^{ \pm}=\left(\nu \times \mathbf{E}_{0}\right)^{ \pm} & \text {on } \Gamma \\
(\nu \times \operatorname{curl} \mathbf{E})^{ \pm}=\left(\nu \times \operatorname{curl} \mathbf{E}_{0}\right)^{ \pm} & \text {on } \Gamma .
\end{aligned}
$$

From Theorem 4.2, if $k$ is purely imaginary with large enough modulus, then (5.1)-(5.4) in $D$ only has trivial solutions. Since $N=n I$, where $n$ is a constant in $\mathbb{R}^{3} \backslash \bar{D}$, then the variational formulation of (5.1)-(5.4) in $\mathbb{R}^{3} \backslash \bar{D}$ is (2.24) where the right hand is 0 and $\mathbb{R}^{3} \backslash \Gamma$ is replaced by $\mathbb{R}^{3} \backslash \bar{D}$. Then $\mathbf{U}=0$, and hence $\mathbf{E}=0, \mathbf{E}_{0}=0$ in $\mathbb{R}^{3} \backslash \Gamma$. The jump relations (2.15)-(2.18) yield $\mathbf{M}=0$ and $\mathbf{J}=0$, and this proves the lemma.

Finally, combining Lemmas 3.2 and 5.1 , we can immediately prove our main theorem using the analytic Fredholm theory [11].

Theorem 5.2. Assume that $N$ satisfies Assumption 3.2.1. Then the set of the transmission eigenvalues in $\mathbb{C}$ is discrete. 
Acknowledgments. S. Meng greatly acknowledges the hospitality of the DeFI Team at Ecole Polytechnique.

\section{REFERENCES}

1. A.S. Bonnet-BenDhia, L. Chesnel and H. Haddar, On the use of $t$-coercivity to study the interior transmission eigenvalue problem, C.R. Acad. Sci. 340 (2011).

2. F. Cakoni and D. Colton, A qualitative approach to inverse scattering theory, Springer, Berlin, 2014.

3. F. Cakoni, D. Colton and P. Monk, The linear sampling method in inverse electromagnetic scattering, CBMS-NSF 80, SIAM Publications, 2011.

4. F. Cakoni, D. Colton, P. Monk and J. Sun, The inverse electromagnetic scattering problem for anisotropic media, Inv. Prob. 26 (2010), 074004.

5. F. Cakoni, D. Gintides and H. Haddar, The existence of an infinite discrete set of transmission eigenvalues, SIAM J. Math. Anal. 42 (2010), 237-255.

6. F. Cakoni and H. Haddar, A variational approach for the solution of the electromagnetic interior transmission problem for anisotropic media, Inv. Prob. Imaging 1 (2007), 443-456.

7. , Transmission eigenvalues in inverse scattering theory, Inv. Prob. Appl., Inside Out 60, MSRI Publications, 2012.

8. _ Transmission eigenvalues, Inv. Prob. 29 (2013), 100201.

9. F. Cakoni and A. Kirsch, On the interior transmission eigenvalue problem, Int. J. Comp. Sci. Math. 3 (2010), 142-167.

10. L. Chesnel, Interior transmission eigenvalue problem for Maxwell's equations: The T-coercivity as an alternative approach, Inv. Prob. 28 (2012), 065005.

11. D. Colton and R. Kress, Inverse acoustic and electromagnetic scattering theory, 3rd edition, Springer, New York, 2013.

12. D. Colton and S. Meng, Spectral properties of the exterior transmission eigenvalue problem, Inv. Prob. 30 (2014), 105010.

13. A. Cossonnière, Transmission eigenvalues in electromagnetic scattering, Ph.D. thesis, CERFACS, Toulouse, France, 2011.

14. A. Cossonnière and H. Haddar, The electromagnetic interior transmission problem for regions with cavities, SIAM J. Math. Anal. 43 (2011), 1698-1715.

15. Surface integral formulation of the interior transmission problem, J. Integral Equations Appl. 25 (2013), 1123-1138.

16. H. Haddar, The interior transmission problem for anisotropic Maxwell's equations and its applications to the inverse problem, Math. Meth. Appl. Sci. 27 (2004), 2111-2129.

17. G. Hsiao and W. Wendland, Boundary integral equations, Springer, Berlin, 2010 .

18. A. Kirsch, An integral equation for Maxwell's equations in a layered medium with an application to the factorization method, Journal of Integral Equations Appl. 19 (2007), 333-357. 
19. A. Kirsch, On the existence of transmission eigenvalues, Inv. Prob. Imaging 2 (2009), 155-172.

20. A. Kirsch and F. Hettlich, The mathematical theory of time-harmonic Maxwell's equations, 190, Springer, Berlin 2015.

21. A. Kleefeld, A numerical method to compute interior transmission eigenvalues, Inv. Prob. 29 (2013), 104012.

22. R. Kress, Linear integral equations, 3rd ed., Springer Verlag, New York, 2014 .

23. E. Lakshtanov and B. Vainberg, Ellipticity in the interior transmission problem in anisotropic media, SIAM J. Math. Anal. 44 (2012), 1165-1174.

24. , Applications of elliptic operator theory to the isotropic interior transmission eigenvalue problem, Inv. Prob. 29 (2013), 104003.

25. W. McLean, Strongly elliptic systems and boundary integral equations, Cambridge University Press, Cambridge, 2000.

26. J.C. Nédélec, Acoustic and electromagnetic equations. Integral representations for harmonic problems, Springer-Verlag, New York, 2001.

27. L. Päivärinta and J. Sylvester, Transmission eigenvalues, SIAM J. Math. Anal. 40 (2008), 738-753.

28. L. Robbiano, Spectral analysis of the interior transmission eigenvalue problem, Inv. Prob. 29 (2013), 104001.

29. J. Sylvester, Discreteness of transmission eigenvalues via upper triangular compact operator, SIAM J. Math. Anal. 44 (2012), 341-354.

Department of Mathematics, Rutgers University, Piscataway, NJ 08854

Email address: fiora.cakoni@rutgers.edu

inRia, Ecole Polytechnique, Université Paris Saclay, Route de Saclay, 91128, Palaiseau, France

\section{Email address: Houssem.Haddar@inria.fr}

Department of Mathematical Sciences, University of Delaware, Newark, Delaware 19716

Email address: sxmeng@udel.edu 